\title{
CHROMOSOME PAIRING IN ALLOHEXAPLOID WHEAT var. CHINESE SPRING. TRANSFORMATION OF MULTIVALENTS INTO BIVALENTS, A MECHANISM FOR EXCLUSIVE BIVALENT FORMATION
}

by

\author{
PALLE HOBOLTH \\ Department of Physiology, Carlsberg Laboratory. \\ Gamle Carlsberg Vej 10, DK-2500 Copenhagen Valby
}

Keywords: Synaptonemal complex, recombination nodule, multivalent, centromere

\begin{abstract}
Meiosis in allohexaploid wheat, Triticum aestivum var. Chinese Spring has been investigated by complete three dimensional reconstructions of a late zygotene and an early pachytene nucleus. At zygotene, specific synapsis of homologous chromosomes and chromosome segments gives rise to extensive multivalent formation with shifts of pairing partners between apparently homoeologous chromosomes. These observations were confirmed by the identification of shifts of pairing partners in three serial sectioned, but only partly reconstructed zygotene nuclei. In striking contrast, the pairing at early pachytene was exclusively in bivalents. These findings, which are consistent with the pairing pattern described in tri- and tetraploid Bombyx oocytes, definitely exclude a presynaptic alignment as the primary cause for a regular bivalent formation in wheat. Instead, multivalents form during the specific zygotene pairing but are later transformed into bivalents through dissolution and reassembly of synaptonemal complexes. It is proposed that the regular bivalent formation in allohexaploid wheat is due to a temporal delay of crossing over until the pairing correction is completed at early pachytene. This temporal delay may be mediated by the action of the $\mathrm{Ph}$ gene(s) on chromosome SB. Finally, interlockings were revealed in the completely reconstructed late zygotene nucleus but were resolved prior to early pachytene.
\end{abstract}

\section{INTRODUCTION}

The common bread wheat, Triticum aestivum, is an allohexaploid $(2 n=6 x=42)$ consisting of three different genomes $\mathrm{A}, \mathrm{B}$ and $\mathrm{D}$. Breeding experiments have suggested that the $A$ and $\mathrm{D}$ genomes originate from $\mathrm{T}$. monococcum and $T$. tauchii respectively whereas the donor of the B genome is not yet unequivocally identified (for reviews see 32, 36). Genetic and cytological investigations have demonstrated that $\mathrm{T}$. aestivum behaves as a normal diploid: It has disomic inheritance, 21 bivalents are present at diakinesis-metaphase I, and in euhaploids, chiasma formation is virtually absent $(30,36)$. Hence, 
crossing over and chiasma formation are restricted to homologous chromosomes within each of the $\mathrm{A}, \mathrm{B}$ and $\mathrm{D}$ genomes.

A number of investigations have, however, shown that considerable similarity exists between the A, B and D genomes. Firstly, as shown by Sears (35), the effect of a lack of one pair of homologues can be compensated for by addition of two extra doses of chromosomes from a different genome. Such nullisomic tetrasomic tests have revealed seven groups of so-called homoeologous chromosomes (partly homologous), each group consisting of 2 homologues from each of the A, B and D genomes. Secondly, crossing over and chiasma formation between chromosomes belonging to the $\mathrm{A}$ and $\mathrm{D}$ genomes have been demonstrated in diploid hybrids between the $\mathrm{A}$ and $\mathrm{D}$ progenitors of hexaploid wheat (20). This clearly shows that sufficient homology exists to permit crossing over and chiasma formation, at least between the $\mathrm{A}$ and $\mathrm{D}$ genomes.

The suppression of crossing over and chiasma formation between the homoeologous chromosomes in $T$. aestivum has been shown to be controlled by several genes (reviewed in 36 ), the most effective one being the $\mathrm{Ph}$ gene(s) located on the long arm of chromosome 5B. In plants, where both copies of the long arm of chromosome 5B are lacking, chiasma formation is no longer restricted to homologous chromosomes as evidenced by the presence of multivalents at metaphase I. By crossing plants nullisomic for chromosome $5 \mathrm{~B}$ with euhexaploid plants, RILEY and KempanNa (31) obtained plants which were heterozygous for translocations. By subsequent crossing to different lines in which each chromosome of the complement in turn was marked by being telocentric Riley and KempanNa (31) were able to identify the chromosomes involved in the translocation and could thereby demonstrate that the initial crossover in the $5 \mathrm{~B}$ nullisomic plant had occurred between homoeologous chromosomes. These results are in agreement with the observation (30) that in polyhaploid plants with 21 chromosomes and deficient for the long arm of chromosome 5B, chiasma formation between homoeologous chromosomes gives rise to bivalents and trivalents at metaphase I.

The effect of additional copies of the long arm of chromosome $5 \mathrm{~B}$ on chiasma formation was analysed by Feldman (9). The presence of four copies of 5BL causes a minor reduction of the number of chiasmata whereas six doses dramatically reduce the number of chiasmata resulting in a decrease in the number of bivalents and a corresponding increase in the number of univalents. On the basis of this experimental evidence, FELdMaN (9) proposed that the gene(s) on the long arm of chromosome 5B somehow mediates the spatial distribution of the chromosomes prior to the meiotic prophase in the following way: In the presence of two doses of 5BL the homologous chromosomes are located closer to each other than the homoeologous chromosomes of the same group and pairing is exclusively in bivalents. In the absence of 5BL, both homologous and homoeologous chromosomes of each group are close together resulting in multivalent formation. Finally, six doses of 5BL suppress premeiotic association entirely causing a random distribution of all chromosomes and hence failure of pairing and chiasma formation.

In an attempt to provide experimental evidence for this proposal, FeldMaN et al. (10) examined the distance between homologous chromosomes in root-tip cells, and concluded that homologous chromosomes were closely associated. Later investigations (11) indicated that also homoeologous chromosomes were associated in root-tip cells and at metaphase I, although to a lesser extent than homologous chromosomes. These conclusions were, however, questioned by DARVEY and DRISColl (8) who failed to find an association of homologous chromosomes at mitotic interphase and metaphase. Furthermore, a number of investigations in both plants and animals have clearly shown that the homologous chromosomes appear to be randomly distributed in the nucleus prior to synapsis and synaptonemal complex formation (28).

The aim of the present investigation is to analyze chromosome pairing and synaptonemal complex formation in order to determine whether chromosome pairing during zygotene in allohexaploid wheat is exclusively in the form of bivalents as proposed by Feldman (9), or whether the pairing, as is the case in tri- and tetraploid Bombyx $(25,27$; see also section 4.1$)$ includes a transient phase of multivalent formation, followed by a transformation of multivalents into bivalents. A further aim is to examine 
the number and distribution of recombination nodules in zygotene and pachytene in order to elucidate the role of crossing over in the exclusive bivalent formation in wheat.

\section{MATERIALS AND METHODS}

The present analysis was performed on normal allohexaploid wheat, Triticum aestivum, variety Chinese Spring. The plants, kindly provided by Dr. J. LARSEN, Carlsberg Laboratory, were grown in a green house at $20^{\circ} \mathrm{C}$ in continuous light. Tillers at the desired stage of development were collected and placed in distilled water. The spike was then removed from the tiller and immersed in $4 \%$ glutaraldehyde in $0.08 \mathrm{M}$ PIPES (piperazine- $\mathrm{N}, \mathrm{N}$ '-bis[2-ethane sulfonic acid]) buffer ( $\mathrm{pH}$ 8.0) (34). One anther from each floret was fixed in 1:3 glacial acetic acid:ethanol for light microscopy, while the other two anthers were fixed in $4 \%$ glutaraldehyde (purification index about 0.2 ) in $0.08 \mathrm{M}$ PIPES buffer ( $\mathrm{pH} 8.0$ ) for $1 \frac{1}{2}$ hours at room temperature, then cut in half and fixed for another 2 hours. Following several washes in 0.2 $\mathrm{M}$ PIPES buffer (pH 6.8), the anthers were postfixed in $2 \% \mathrm{OsO}_{4}$ in $0.18 \mathrm{M}$ PIPES buffer (pH 6.8) for $3 / 4$ hour at room temperature, washed twice in buffer, 3 times in distilled water and postfixed/stained in a $2 \%$ aqueous uranyl acetate solution at room temperature for $3 \frac{1}{2}$ hours. After two washes in distilled water, the anthers were dehydrated in a graded alcohol series, embedded in Spurr's low viscosity resin, and polymerized for 24 hours at $70^{\circ} \mathrm{C}$. Thick sections of the anthers were examined in the light microscope for a preliminary identification of meiotic stages. Nuclei at the desired stage were serially sectioned on a Reichert Om U3 ultramicrotome, equipped with a diamond knife. The sections were double stained in $5 \%$ aqueous uranyl acetate for 30 minutes at $60^{\circ} \mathrm{C}$ and in lead citrate for 20 minutes at room temperature in an LKB Ultrostainer 2168, System Carlsberg. The sections were analyzed in a Siemens 102 electron microscope at $80 \mathrm{kV}$. Micrographs of the selected nuclei were taken at a primary magnification of 3000 times, and printed to a final magnification of 11,000 to 15,600 times.

The three dimensional reconstructions of the synaptonemal complexes and the lateral compo- nents, as well as the length measurements, were performed as described by RASMUSSEN and HOLM (26).

In the present study five nuclei, all from the same plant, were serially sectioned and photographed, and two of them were reconstructed completely.

\section{RESULTS}

\subsection{Stage determination}

Light microscopical examination of squashed, orcein stained meiotic nuclei, including nuclei from the same plant as that used for the ultrastructural analysis, revealed a sequence of stages identical to that described in earlier reports on the normal progression of meiosis in hexaploid wheat $(2,3,33)$. Only bivalents were present at metaphase I in all analyzed plants and the disjunction of the homologous chromosomes at anaphase I appeared regular. Hence, neither the genetic constitution of the plants nor the condition under which they were grown appeared to induce multivalent formation.

The criteria used to establish the temporal sequence of the serially sectioned nuclei are similar to those generally used at the electron microscopic level $(28,37): 1)$ The leptotene stage is characterized by the organization of a lateral component in each chromosome. 2) The initiation of the zygotene stage is marked by the appearance of the first pieces of synaptonemal complex. During zygotene, the telomeres are attached to the nuclear envelope within a restricted area. 3) At pachytene, chromosome synapsis and synaptonemal complex formation are completed. A polarized distribution of the attachment sites of the telomeres persists during the first period of pachytene but is by $\mathrm{mid}$ pachytene no longer observable, the telomeres being more or less uniformly distributed throughout the nuclear envelope.

Following these criteria, two of the analyzed nuclei were in mid zygotene as judged by the presence of both synaptonemal complexes and unpaired lateral components. Two nuclei, of which one was completely reconstructed, exhibited an almost complete synapsis and synaptonemal complex formation, and a prominent aggregation of the attachment sites of the telomeres and were thus classified as being in 


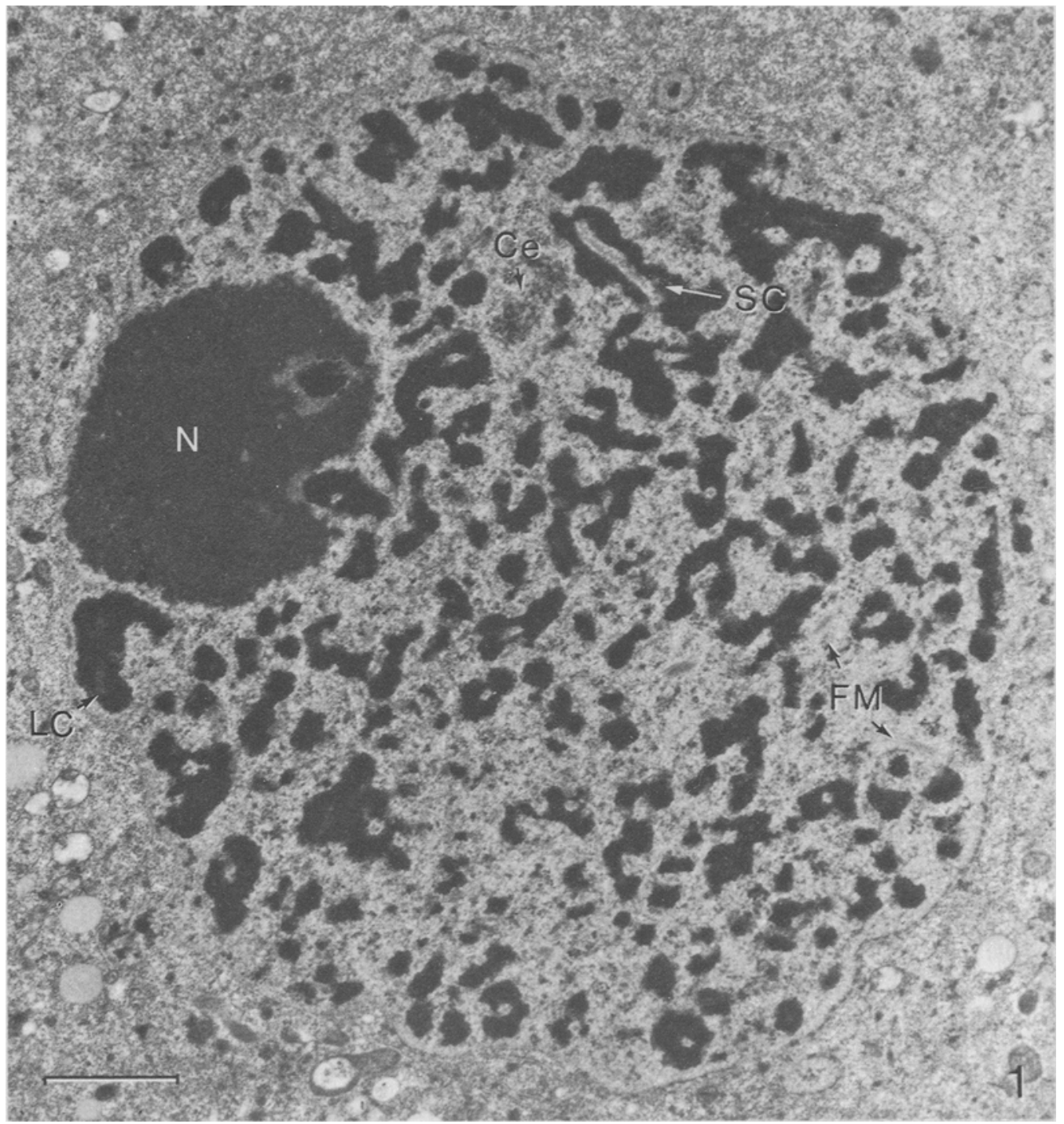

Figure 1. Survey micrograph of a mid zygotene nucleus showing both stretches of unpaired lateral components (LC) and completed synaptonemal complexes (SC).

Note the heavily condensed chromatin, the centromere region $(\mathrm{Ce})$ and the bundles of fibrillar material (FM). $\mathrm{N}$, nucleolus. $(\mathrm{Bar}=2 \mu \mathrm{m})$

late zygotene. In the last nucleus, which was also completely reconstructed, all chromosomes were paired into bivalents, each with a continuous synaptonemal complex from telomere to telomere. The attachment sites of the telomeres in this nucleus were polarized, although to a lesser extent than in the two late zygotene nuclei, hence placing it at early pachytene.
During the period from mid zygotene to early pachytene, the callose wall around the microsporocytes gradually becomes thicker while the chromatin undergoes a progressive decondensation (Figures 1-3). These observations support the temporal sequence described above. 


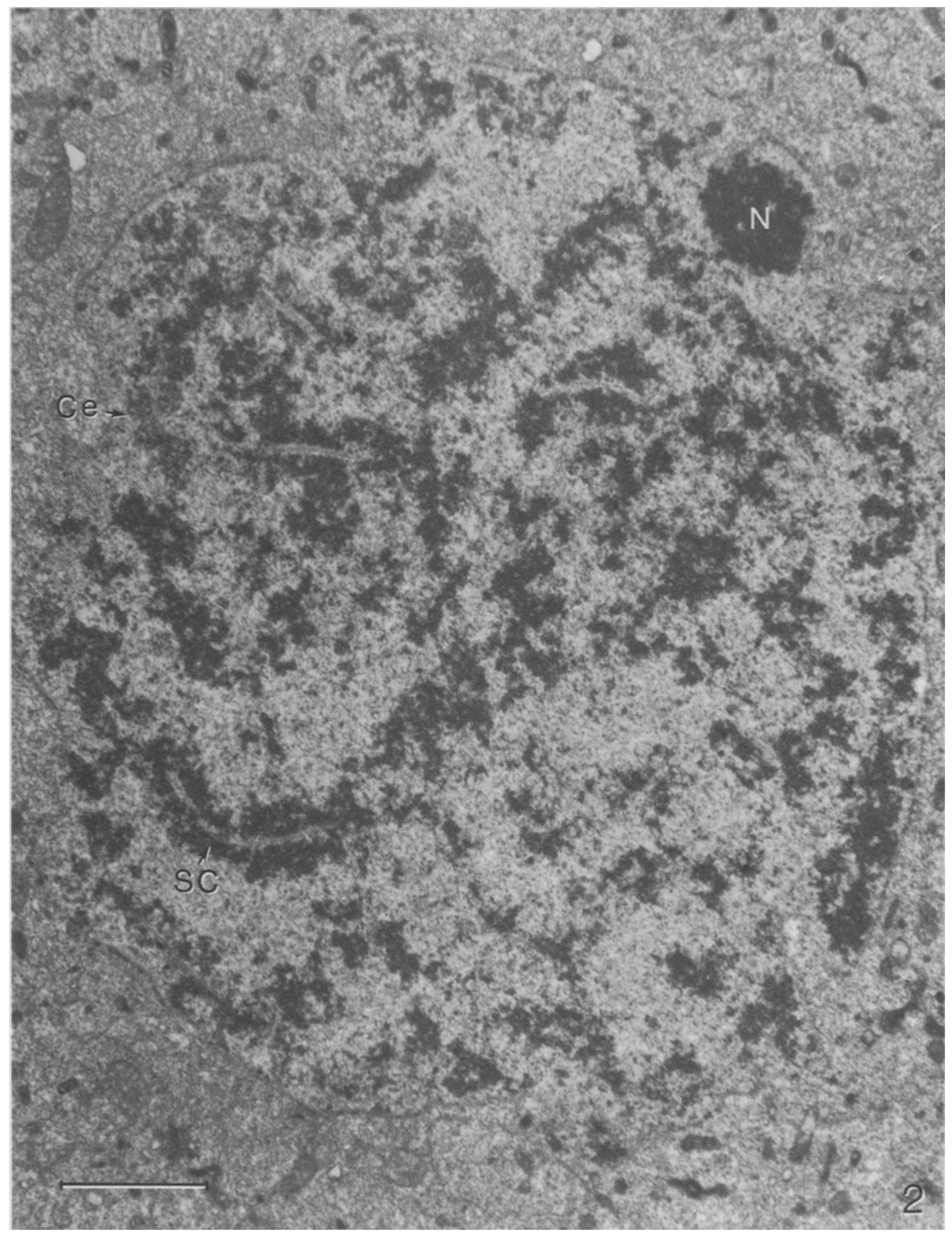

Figure 2. Survey micrograph of a late zygotene nucleus with stretches of synaptonemal complexes (SC).

Note that the chromatin is less condensed than at mid zygotene. Ce, centromere; $\mathrm{N}$, nucleolus. (Bar $=2 \mu \mathrm{m})$. 


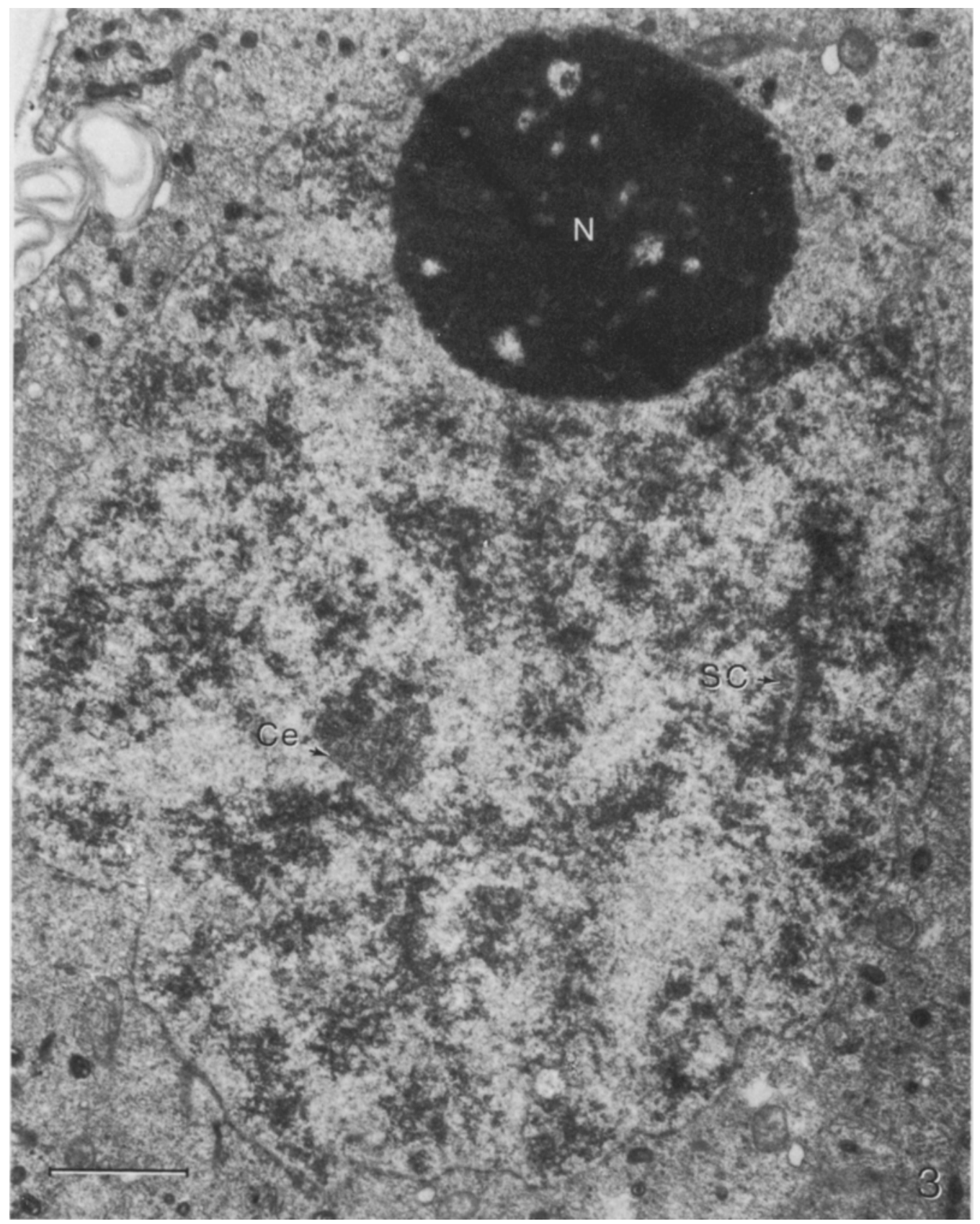

Figure 3. Survey micrograph of an early pachytene nucleus.

The nucleolus $(\mathrm{N})$ is appressed towards the nuclear envelope. The chromatin around the synaptonemal complexes (SC) is less condensed than at late zygotene. Ce, centromere. (Bar $=2 \mu \mathrm{m}$ ). 


\subsection{General aspects}

The morphology of the nuclei at mid and late zygotene and early pachytene is illustrated in Figures 1 to 3. As can be seen from the micrographs, a dramatic decondensation of the chromatin occurs between mid zygotene and early pachytene, from the almost electron opaque configuration at mid zygotene (Figure 1) to the relatively decondensed state of the early pachytene chromatin (Figure 3). At all three stages, the centromere regions are recognizable as more or less spherical or elongated masses with an electron density lower than that of the chromatin (Figure 4). Both unpaired lateral components and completed synaptonemal complexes pass uninterrupted through the centromere regions. In the two mid zygotene nuclei, 28 and 30 centromere regions were identified of which 14 and 12 were associated with synaptonemal complexes, while

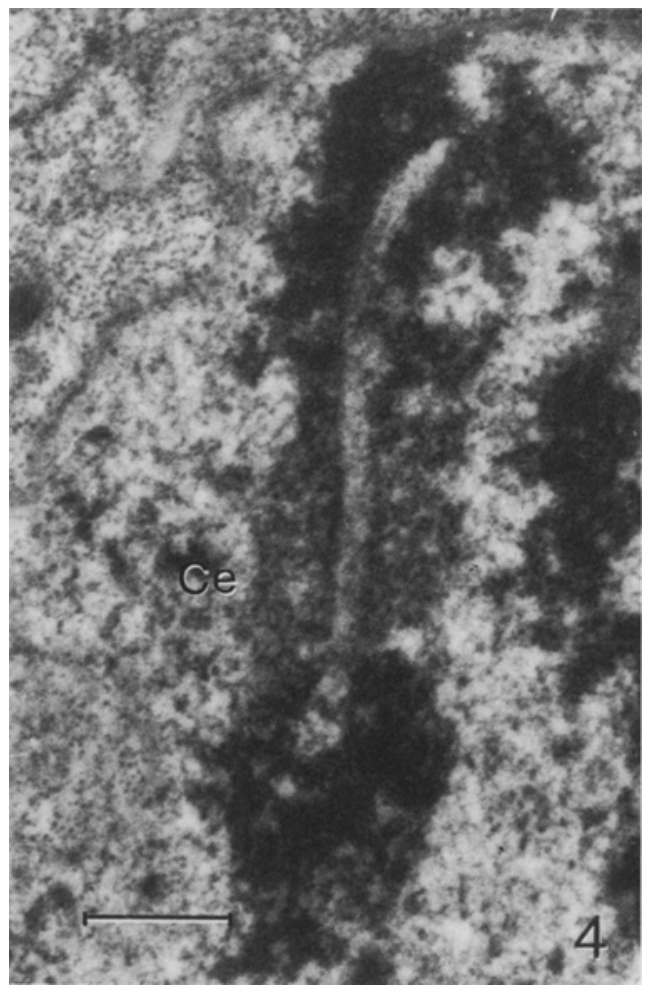

Figure 4. High magnification of synapsed centromere regions at late zygotene.

The synaptonemal complex passes uninterrupted through the centromere region $(\mathrm{Ce}) .(\mathrm{Bar}=0.5 \mu \mathrm{m})$ the remaining 14 and 18 were associated with unpaired lateral components. The number of centromere regions had decreased to 23 and 25 in the two late zygotene nuclei and only 4 and 8 were at this stage unpaired. Both the paired and the unpaired centromere regions appeared to be dispersed at random (Figure 5) within about one half of the nuclear volume (one centromere is on each of sections 23 and 137 while 19 centromeres are between sections 50 and 118 of a total of 148 sections). By early pachytene 21 centromere regions were identified (Figure 5), all associated with synaptonemal complexes. As in late zygotene, the centromere regions at early pachytene appeared to be confined to approximately one half of the nuclear volume (all 21 centromeres are between sections 44 and 114 of a total of 168 sections).

In the completely reconstructed late zygotene nucleus, nucleolus organizing regions were found in four paired chromosome regions, two organizer regions being on chromosome pairs involved in a hexavalent (chromosomes A and B in Figure 18), one on a chromosome pair in a quadrivalent (chromosomes A in Figure 14), and one on chromosomes involved in an association with two fragments (chromosomes A in Figure 20). Three of the organizer regions were associated with the same major nucleolus while the fourth (A in Figure 20) was associated with a minor nucleolus. All four organizer regions appeared similar in ultrastructure consisting of a dense knob and an adjacent, more electron transparent region, the latter being in intimate contact with and partly embedded in the nucleolus (Figure 6). Inside the nucleolus, synaptonemal complex continuity was disrupted and paired chromosomes could not be followed.

In contrast, nucleolus organizing regions were only present in two, possibly three of the bivalents in the early pachytene nucleus (Figures 22 and 27), all associated with the same nucleolus. The longest bivalent was intimately associated with the large nucleolus (Figure 22) but only one of its lateral components appeared to be embedded in the nucleolus. This may be indicative of at least one nucleolus organizing region. The location of an organizer region distally in the long arm of the longest bivalent is, however, at variance with light microscopical determinations of the positions of organizer 


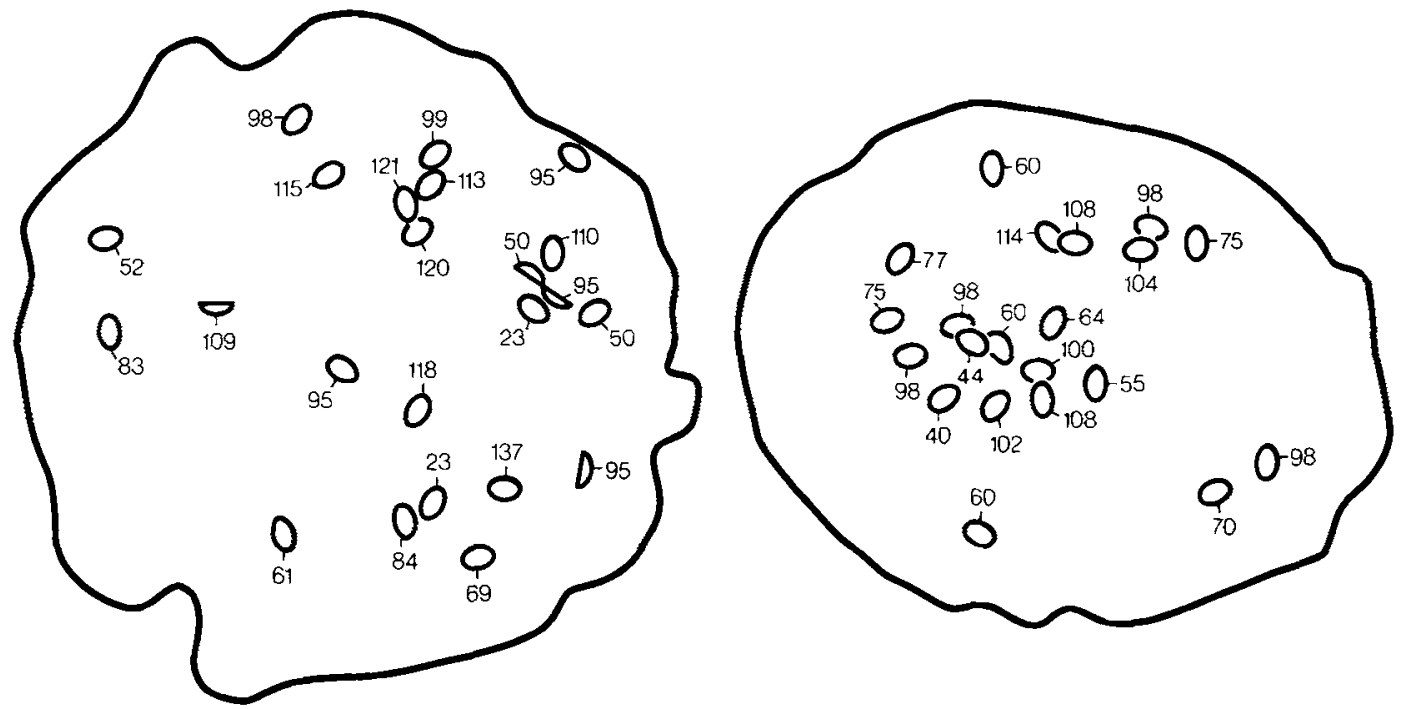

Figure 5. A diagrammatic representation of the position of centromere regions in the late zygotene (left) and the early pachytene nucleus (right).

Single centromere regions associated with only one of the lateral components of the synaptonemal complex are shown as semi-ellipses. The numbers are the section numbers. (Magnification approximately 3,200 times.)

regions in wheat $(4,7)$ and the association of the long arm of bivalent 1 with the nucleolus is most likely incidental. Individual organizer regions could not be identified unequivocally in the two mid zygotene nuclei and the second late zygotene nucleus due to extensive fusion of the nucleolus organizing regions at the periphery of the nucleolus. However, it does seem reasonable to conclude, in agreement with previous light microscopic and biochemical observations $(4,7$, 12 ), that the chromosome complement of wheat contains four pairs of nucleolus organizing regions. The presence of only two pairs of organizer regions in the pachytene nucleus may be due to inactivation of two of the four organizer regions.

Several dense knobs, i. e., spheres of heavily compacted chromatin (Figure 9) were identified in all the analyzed nuclei. In the reconstructed late zygotene nucleus, ten knobs were present of which six were located in telomere regions and four were interstitial. Two of the interstitial knobs were located lateral to the synaptonemal complex while the other two surrounded the complex. In the latter case, the synaptonemal complex was discontinuous inside the knob. In the pachytene nucleus seven knobs were found of which one was telomeric. The remaining six knobs were all interstitial, one being located around the synaptonemal complex while five were located lateral to the complex. As in late zygotene, the synaptonemal complex was discontinuous inside the interstitial knob surrounding the complex. It is thus apparent that dense knobs in wheat vary in position as well as number, and thus are of limited value as morphological chromosome markers.

Figure 6. Five consecutive sections through a nucleolus organizing region.

A reconstruction of the five sections is given in Figure $6 f$ and a complete reconstruction of the chromosome pair is shown in Figure 18 (chromosomes A). Note the dense knob (K) and the electron transparent region (L), the latter penetrating into the nucleolus. The synaptonemal complex (SC) is discontinuous inside the nucleolus. (Bar $=0.5 \mu \mathrm{m})$. 


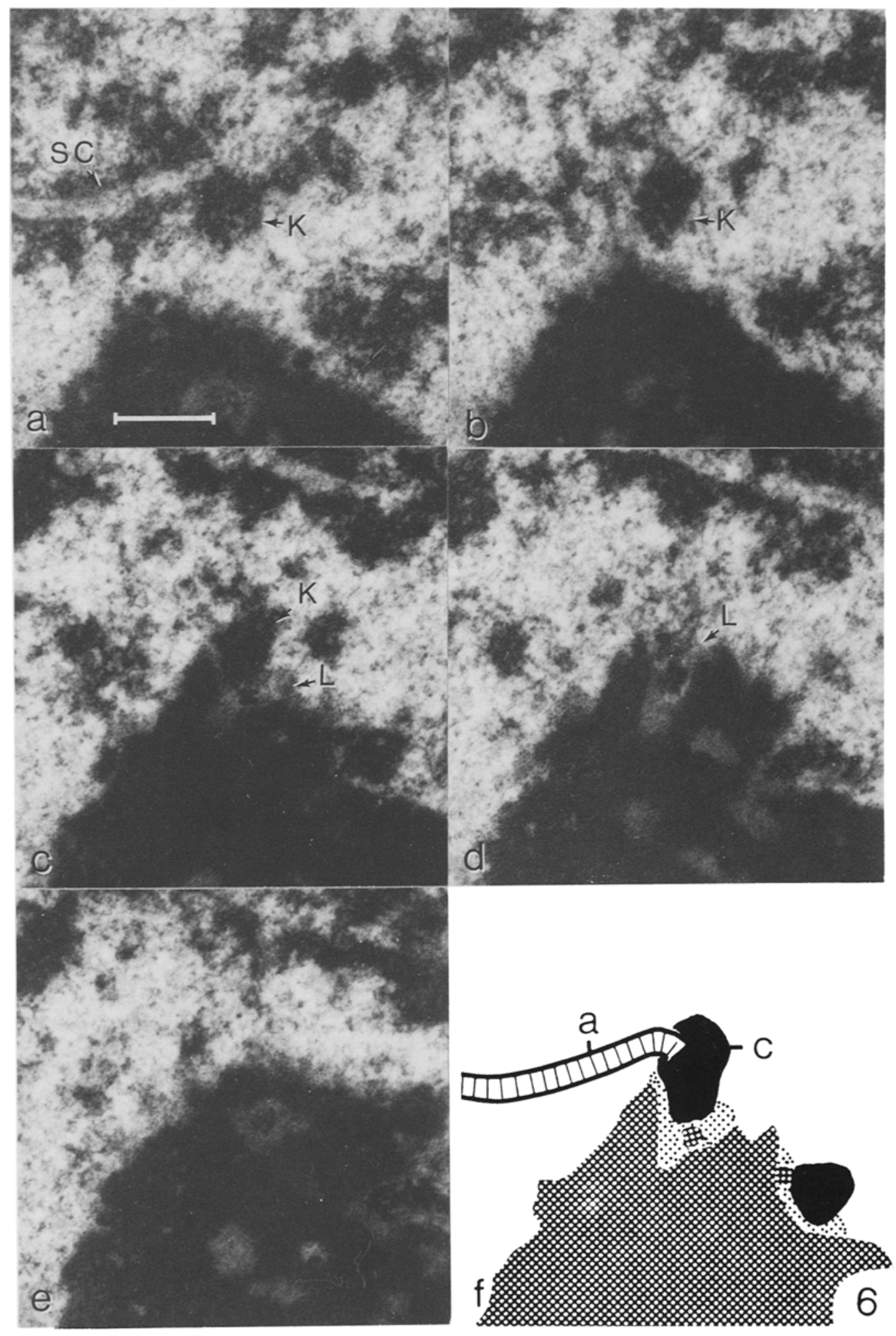




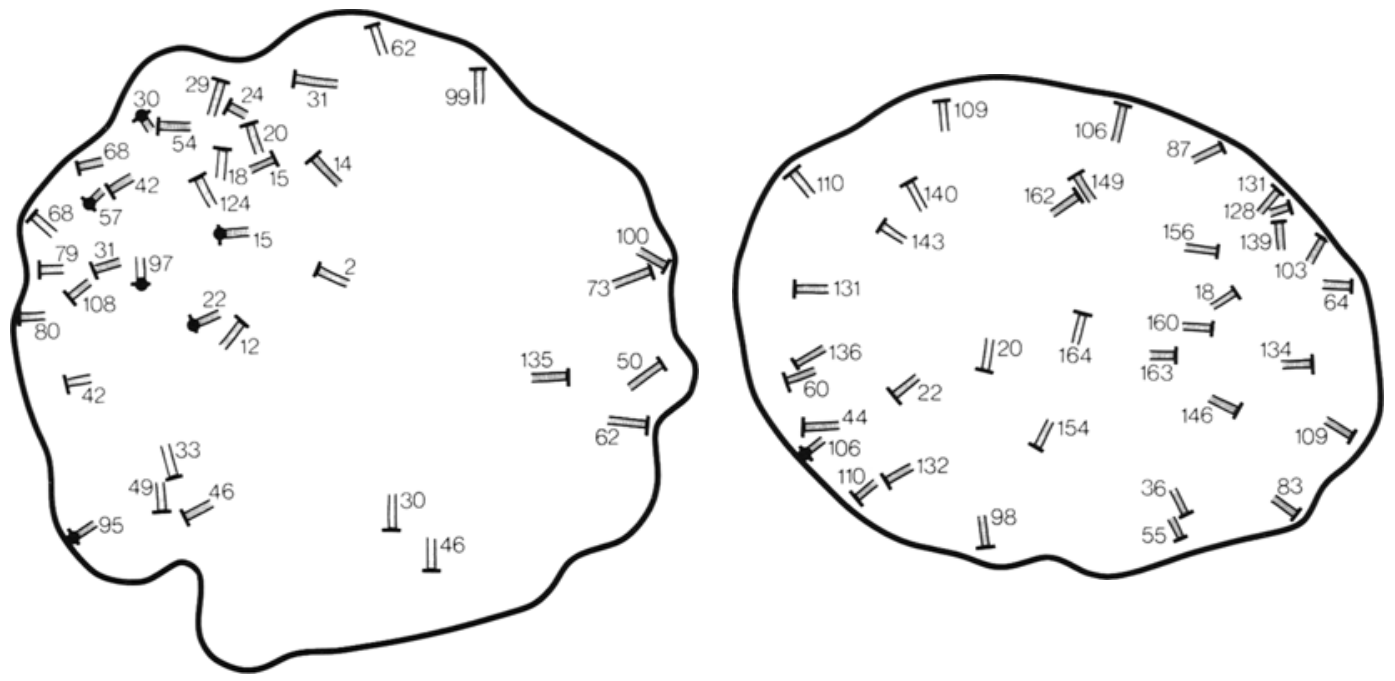

Figure 7. A diagrammatic representation of the attachment sites of the telomeres to the inner membrane of the nuclear envelope at late zygotene (left) and early pachytene (right).

Terminal knobs are shown as filled circles. The numbers are the section numbers. (Magnification approximately 3,200 times)

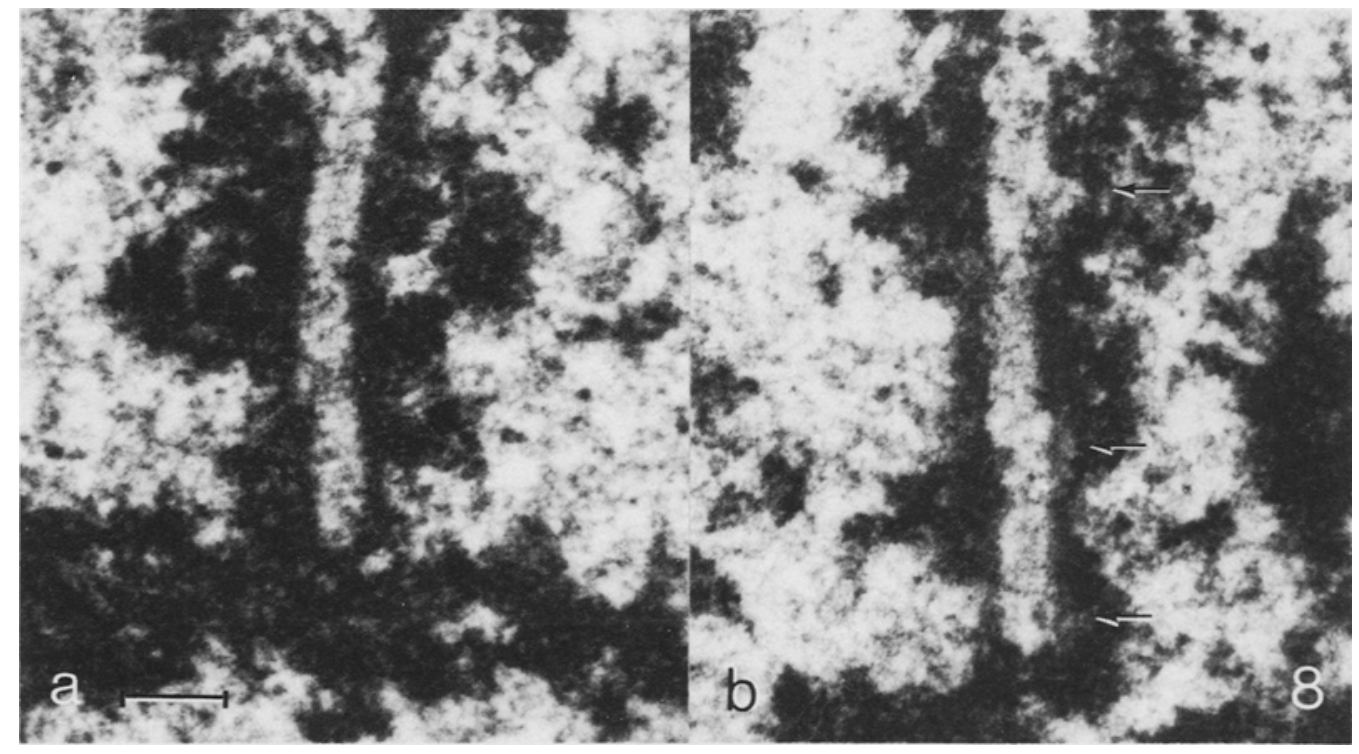

Figure 8. High magnifications of synaptonemal complexes at late zygotene.

Figure 8a shows the regular tripartite structure of the complex as seen in most bivalent regions. In some regions (Figure $8 \mathrm{~b}$ ) the complex is asymmetrical, the lateral components forming small loops (denoted by arrows). (Bar $=0.2 \mu \mathrm{m}$ ).

Figure 9. Six consecutive sections through a shift of pairing partners between one pair of chromosomes, one centric and one acentric fragment (bivalent B and chromosome C in Figure 19).

Each micrograph is accompanied by a graphic interpretation. A reconstruction of the six sections is given in Figure $9 \mathrm{~g}$. $\mathrm{K}$, dense knob. $(\mathrm{Bar}=0.5 \mu \mathrm{m})$. 

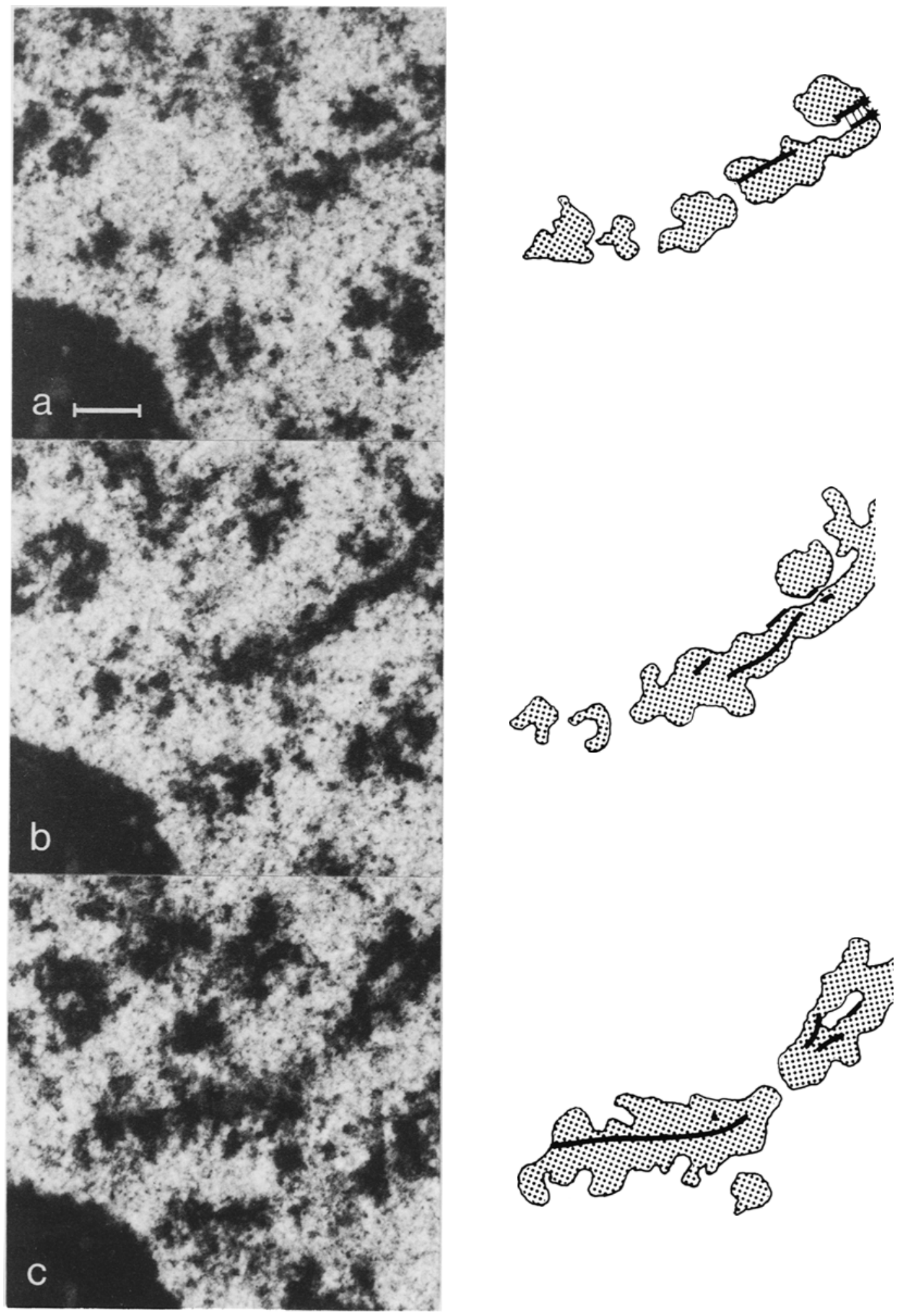


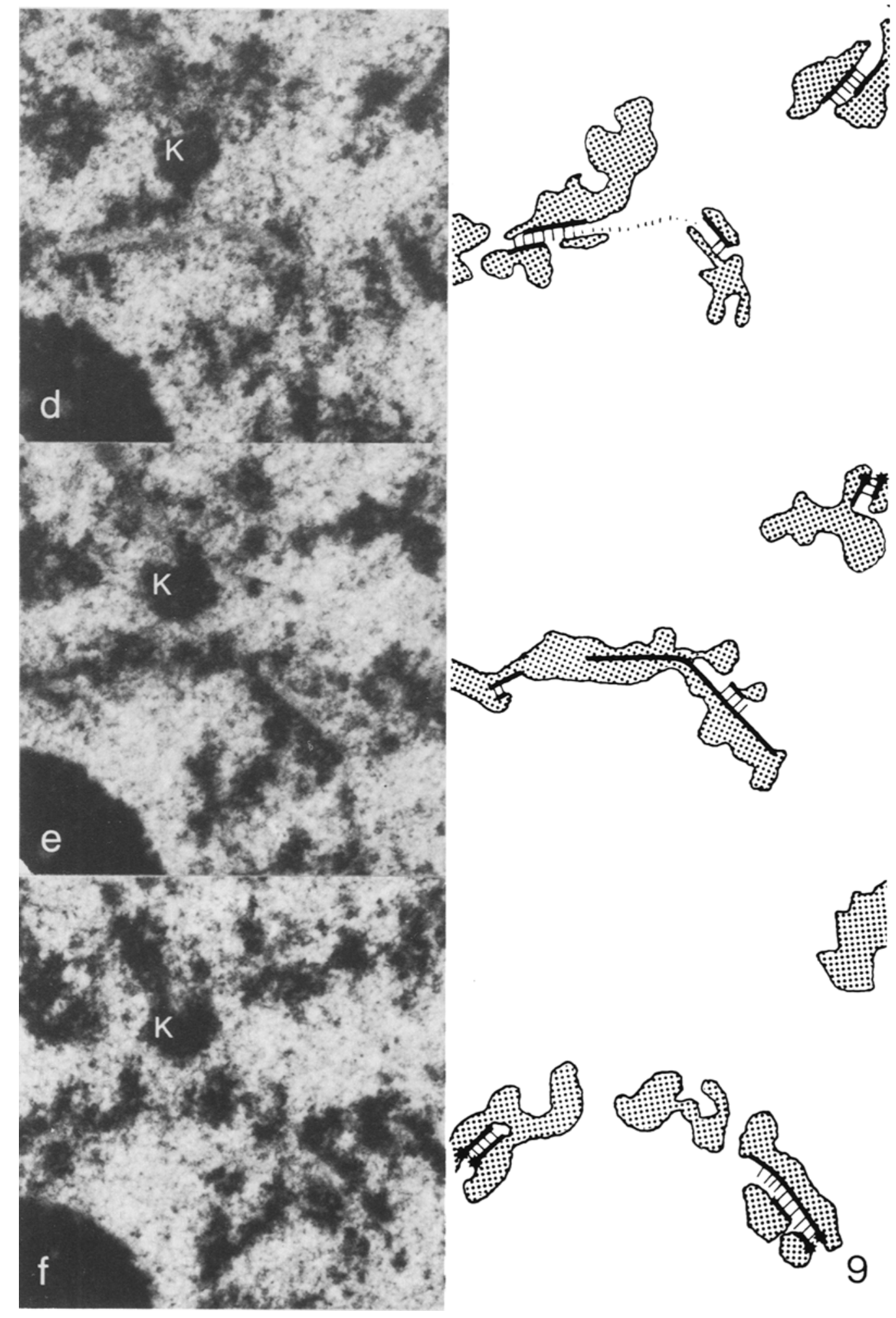




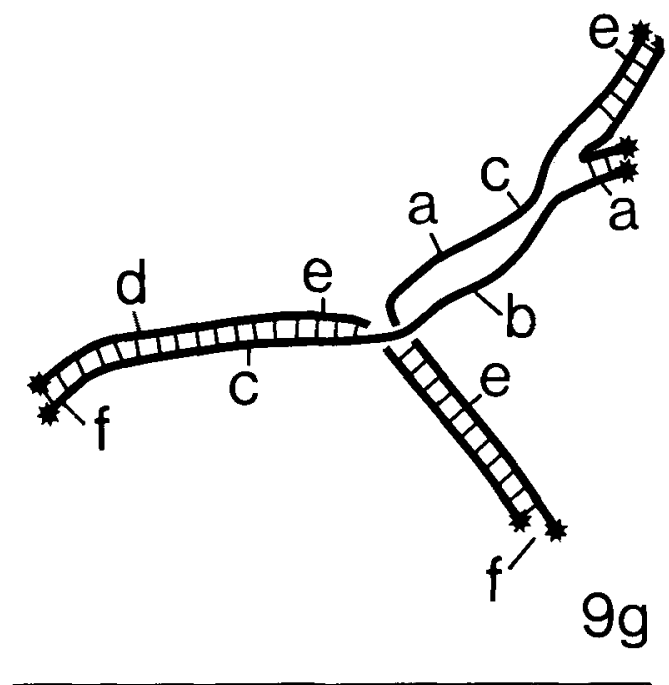

\subsection{Late zygotene}

The complete reconstruction of the late zygotene nucleus revealed that all 84 telomeres were paired two-by-two with a synaptonemal complex. Thirty-seven of the 42 telomere pairs were attached to the inner membrane of the nuclear envelope (Figure 7) while the remaining five were free in the nucleoplasm. The binding of the lateral components to the nuclear envelope occurred in six cases through dense knobs.

The distribution of the attachment sites was clearly polarized, the majority of the attachment sites being concentrated within a limited region of the nuclear envelope (Figure 7). As the length of most of the chromosomes at late zygotene is several times larger than the nuclear diameter, a distinct chromosome bouquet is not evident.

The vast majority of the chromosome complement was paired with synaptonemal complexes leaving only few stretches of lateral components unpaired. Most regions were combined with a synaptonemal complex (Figure 8a) which in ultrastructure and dimensions is similar to that reported from other studies of wheat $(3,21,22)$. In several regions, however, the structure of the complex was less regular (Figure 8b): Frequently, one of the lateral components forms a small loop while at other sites, the chromatin appeared to form a loop, apparently separated from the straight lateral component. Since the identification of such configurations was ambiguous in several cases it was not possible to map the position of the lateral component or chromatin loops along the complex.

The reconstruction of all synaptonemal complexes and lateral components revealed five completely paired bivalents each with a continuous synaptonemal complex from telomere to telomere (Figure 13). Three of the bivalents were attached at both ends to the nuclear envelope, while two were attached at only one end.

The pairing and synaptonemal complex formation of the remainder of the chromosome complement were less regular: Multiple initiation sites for complex formation between different combinations of chromosomes have resulted in extensive multivalent formation, comprising four quadrivalents, a hexavalent, a pentavalent, an association of two chromosomes and two acentric fragments, a centric and an acentric bivalent fragment and a univalent (Figures 1421).

Both in the four quadrivalents and in the hexavalent, one shift of pairing partner is followed by a second shift in the immediate vicinity, resulting in even numbers of shifts between any two pairs of chromosomes, the only exception being the single shift of pairing partners between chromosomes $\mathrm{B}$ and $\mathrm{C}$ in the hexavalent (Figure 18). The series of micrographs in Figure 9 illustrates a single shift of pairing partners in an association of five chromosomes (the shift between chromosomes B and $\mathrm{C}$ in Figure 19).

Although the second late zygotene nucleus was not compietely reconstructed, similar shifts of pairing partners were readily identified (Figure 10). Partial reconstructions of lateral component fragments and chromatin contour clearly indicated the presence of shifts also in the two mid zygotene nuclei. At late zygotene the exact tracing of individual lateral components was hampered by the similarity in electron density of the lateral components and the surrounding chromatin, as well as by the twisting of the synaptonemal complexes. Often one, or more rarely both, lateral components were discontinuous over a short distance at the site of pairing partner exchange. Hence, in most cases it was not possible to establish the course of the individual chromosomes constituting the multivalents by their lateral components alone.

The four quadrivalents in the reconstructed 

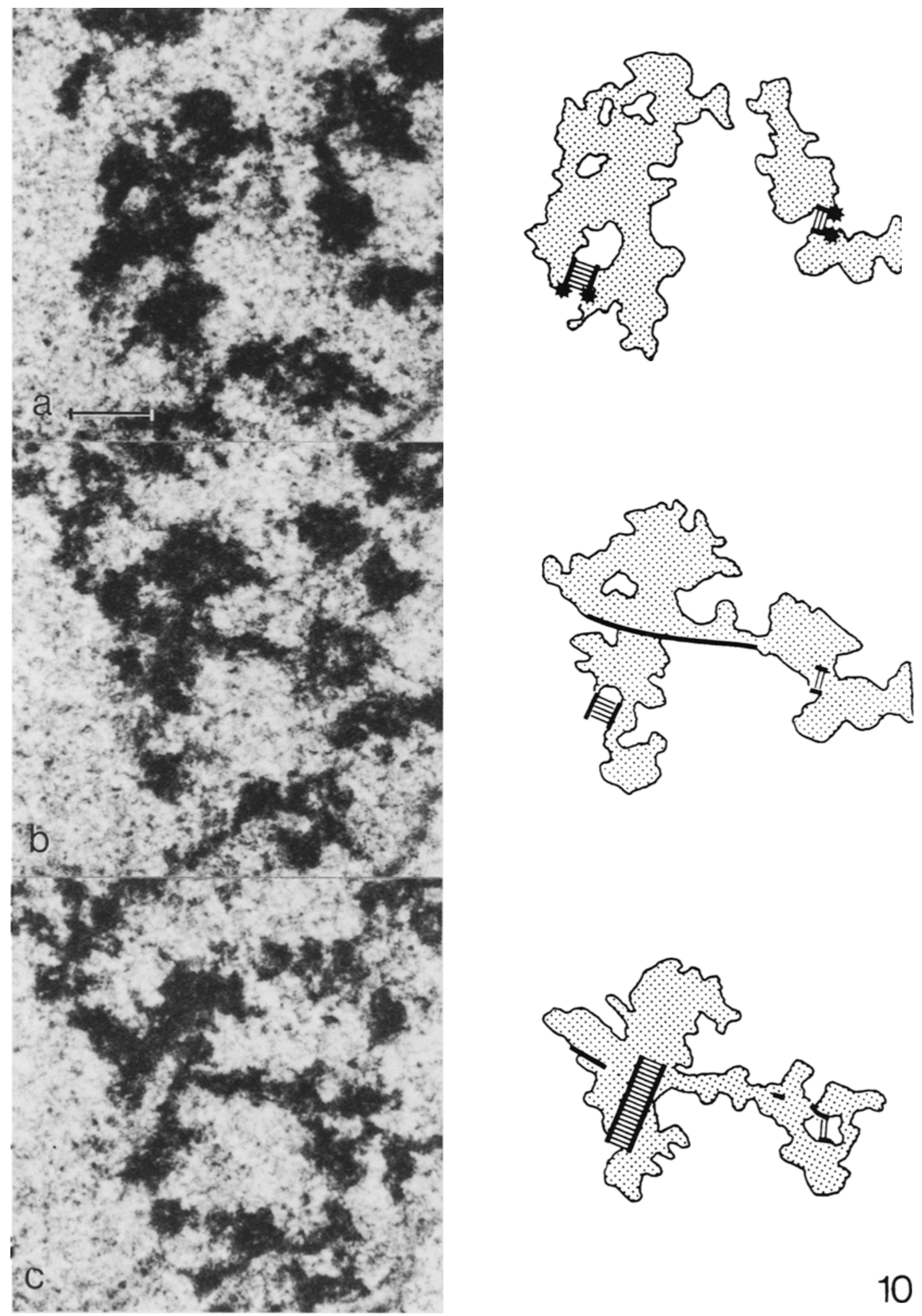


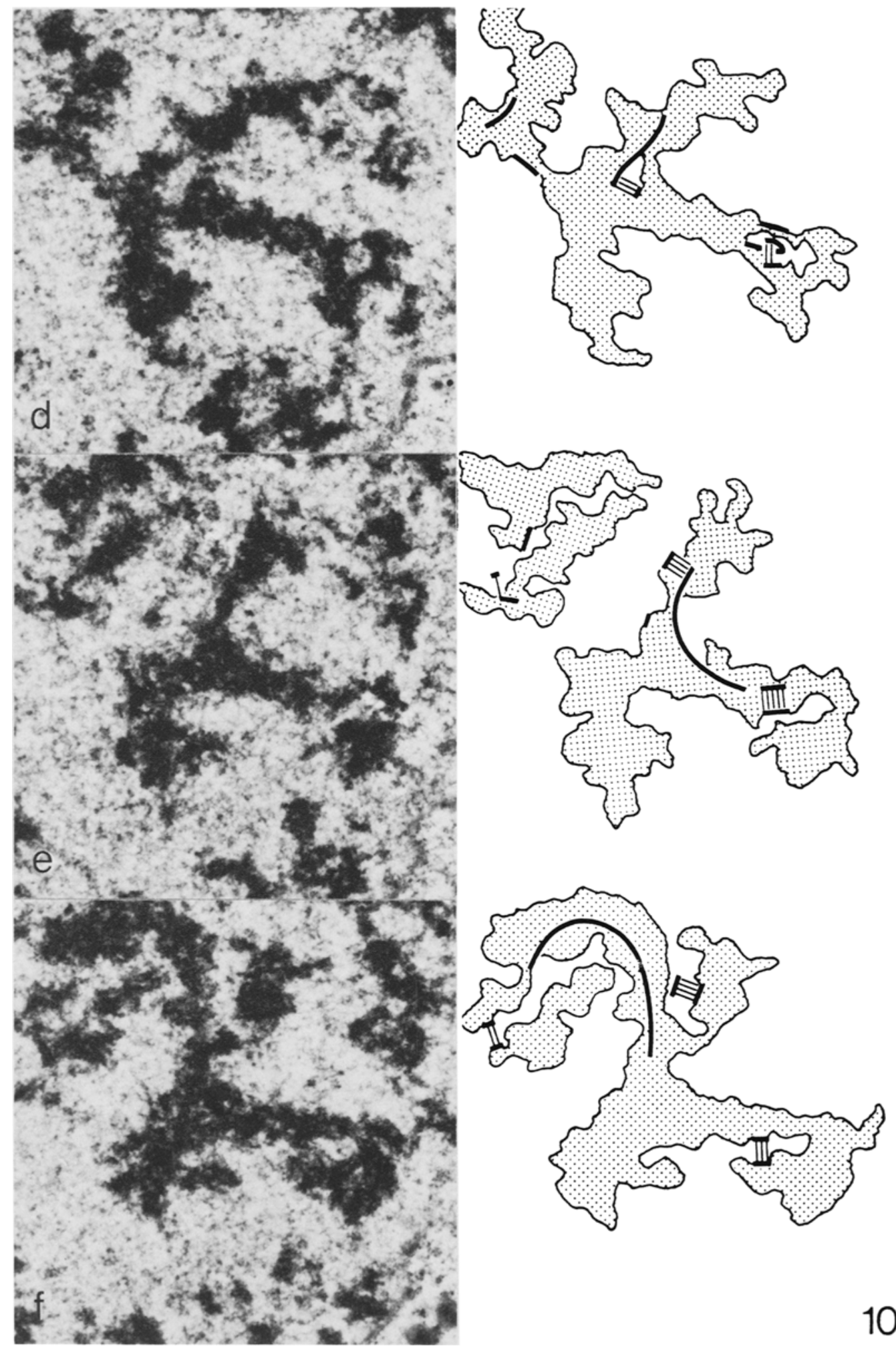




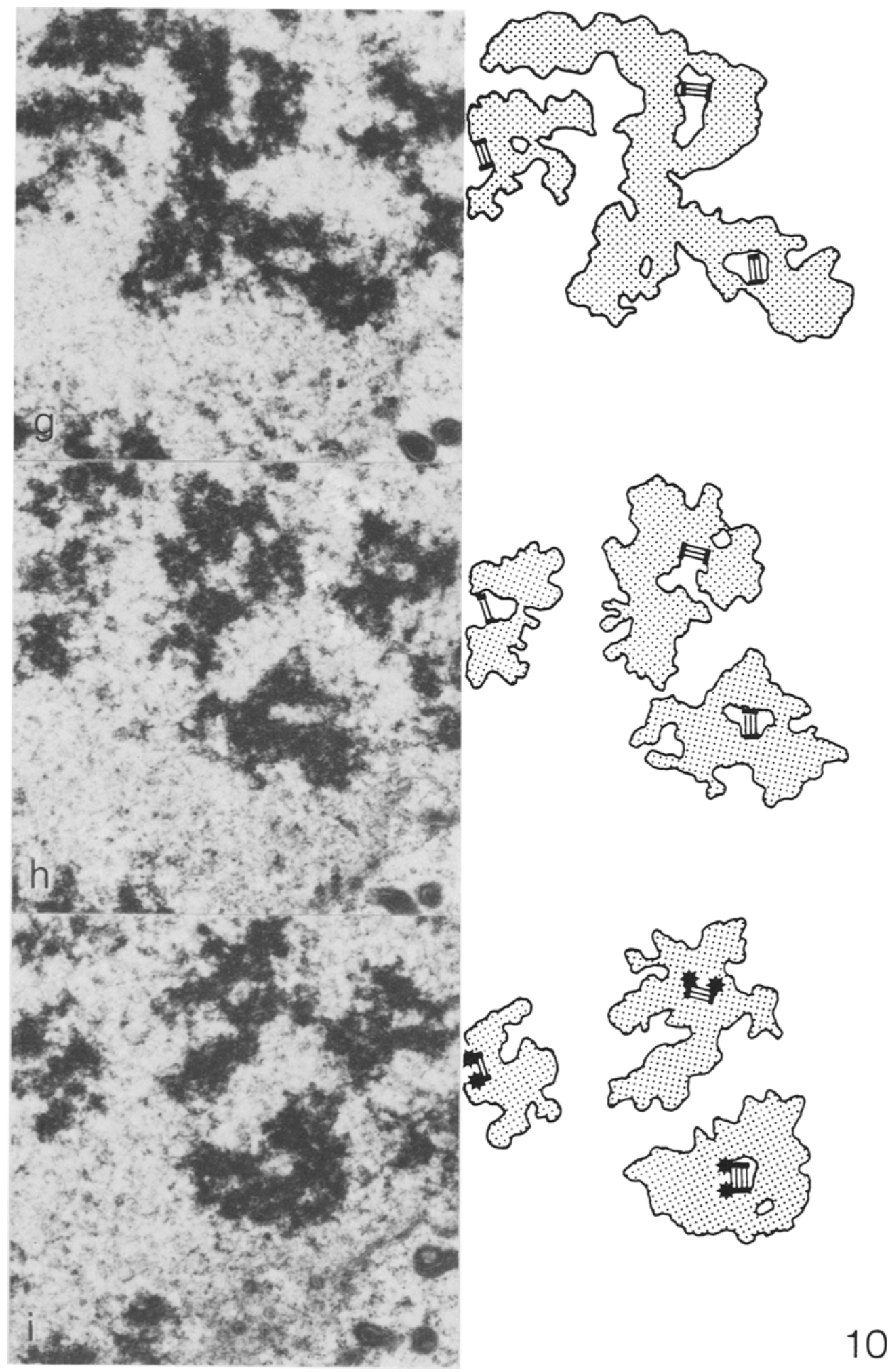




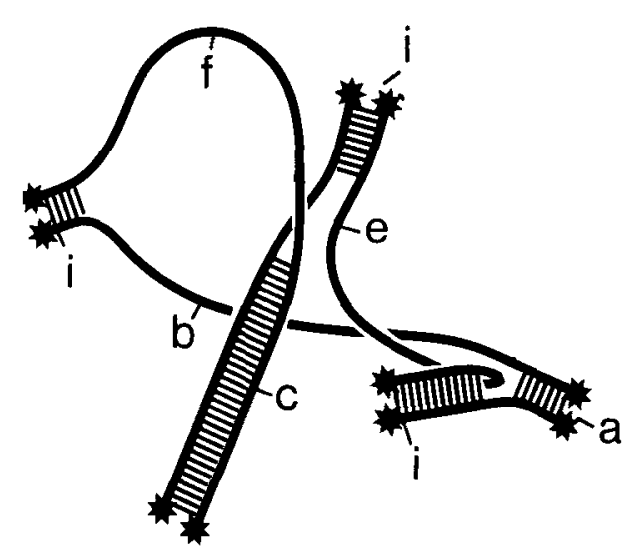

10j

zygotene nucleus are shown in Figures 14 to 17 each accompanied by a diagrammatic representation of the involved chromosomes giving the absolute length as well as the positions of shifts of pairing partners, centromeres, nucleoli etc. Three of the quadrivalents (Figures 14-16) had two shifts of pairing partners whereas the last one had four (Figure 17). In all cases, the shifts of pairing partners were in the vicinity of each other and on the same side of the centromere region. In the hexavalent, five shifts of pairing partners were identified (Figure 18). Four of the shifts were between the A and B chromosomes, two on either side of the nucleolus organizing region and all four on the same side of the centromere region. A single subterminal shift was present between the $B$ and $C$ chromosomes. As shown in the reconstruction and diagram (Figure 18), the two lateral component segments between the $\mathrm{B}$ and $\mathrm{C}$ chromosomes at the site of the shift were paired with a synaptonemal complex. Nucleolus organizing regions have been identified in the light microscope on chromosomes $1 \mathrm{~A}$ and $1 \mathrm{~B}(4,7$, 12 ) and the chromosomes comprising this hexavalent may thus constitute the homoeologous group 1.
Pairing and synaptonemal complex formation were highly irregular in the remainder of the chromosome complement which comprises one association of five chromosomes, one association of two chromosomes and two acentric fragments, a centric and an acentric bivalent fragment and a univalent.

The synaptonemal complex formation in the association of five chromosomes is illustrated by the reconstruction and the diagram in Figure 19. The $A$ and $B$ bivalents have a single subterminal shift of pairing partners. The second shift of pairing partners is also subterminal, but at the opposite end of the B bivalent and involves the synaptonemal complexes designated $\mathrm{C}$. The $\mathrm{C}$ fragments are connected by synaptonemal complexes with two more chromosome fragments giving rise to a very complex configuration consisting of eight synaptonemal complex segments interconnected by unpaired lateral components. As only one centromere region is present in this configuration, and due to the presence of a single univalent in the nucleus (Figure 21) it is likely that these fragments together constitute one chromosome. In contrast to the situation in the four quadrivalents and the hexavalent, only single shifts of pairing partners were found in this pentavalent.

The association depicted in Figure 20 is likewise remarkable: The A chromosome pair, which possesses one of the nucleolus organizing regions and the acentric bivalent fragment $B$ are held together by two shifts of pairing partners. In the A chromosome pair, the two centromere regions are each paired with noncentromeric chromosome regions through a synaptonemal complex as illustrated by the series of micrographs in Figure 11 and the reconstruction in Figure 20. In the other late zygotene nucleus such nonhomologous pairing of centromere regions was also observed.

The remaining chromosomes comprise a univalent, a broken chromosome pair and an acentric fragment of a chromosome pair as shown in Figure 21. The A fragment in Figure

Figure 10. Nine consecutive sections through two shifts of pairing partners between three pairs of chromosomes from the second partially reconstructed late zygotene nucleus.

Each micrograph is accompanied by a graphic interpretation. A reconstruction of the nine sections is given in Figure $10 \mathrm{j}$. $(\mathrm{Bar}=0.5 \mu \mathrm{m})$. 


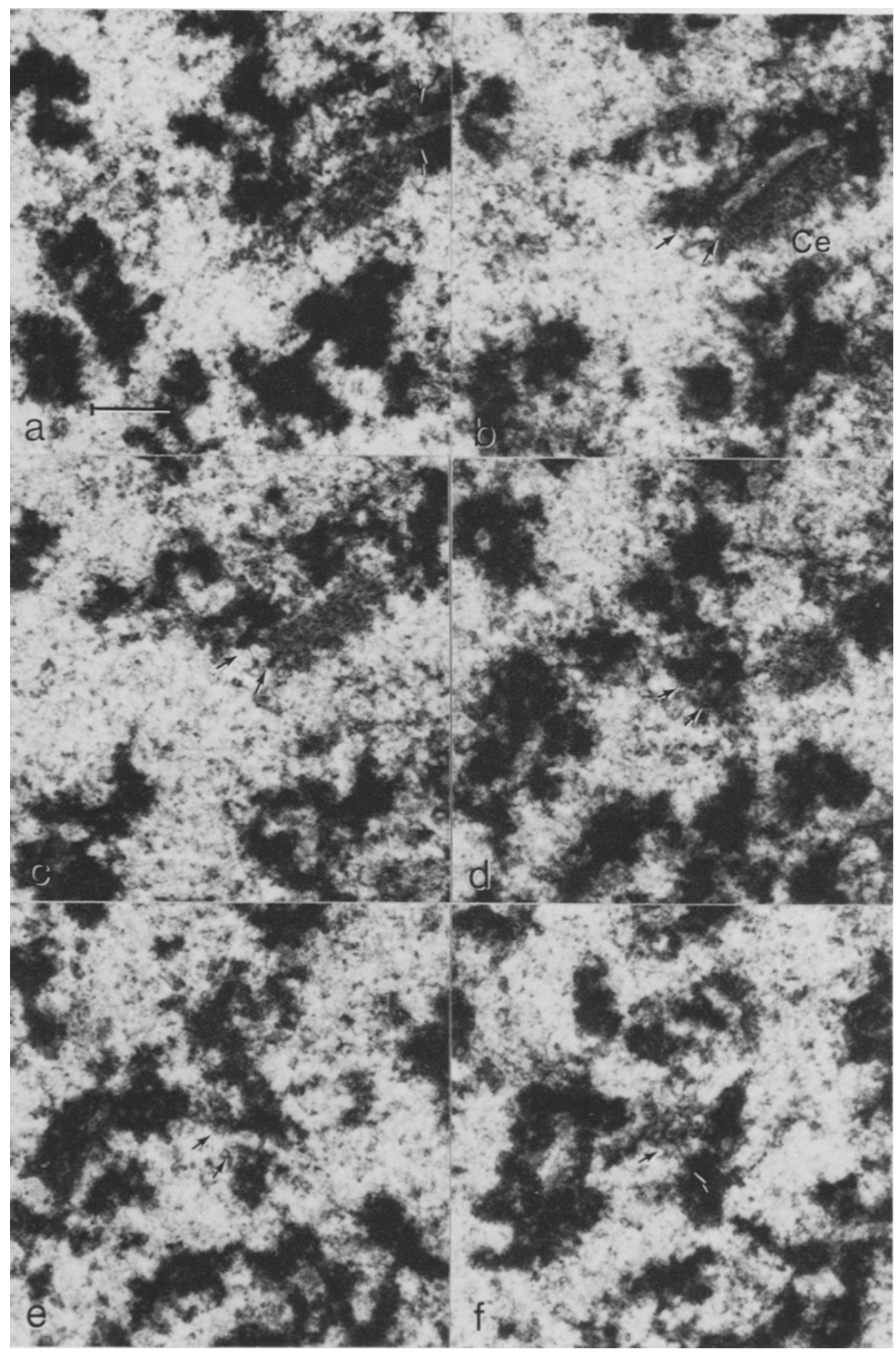




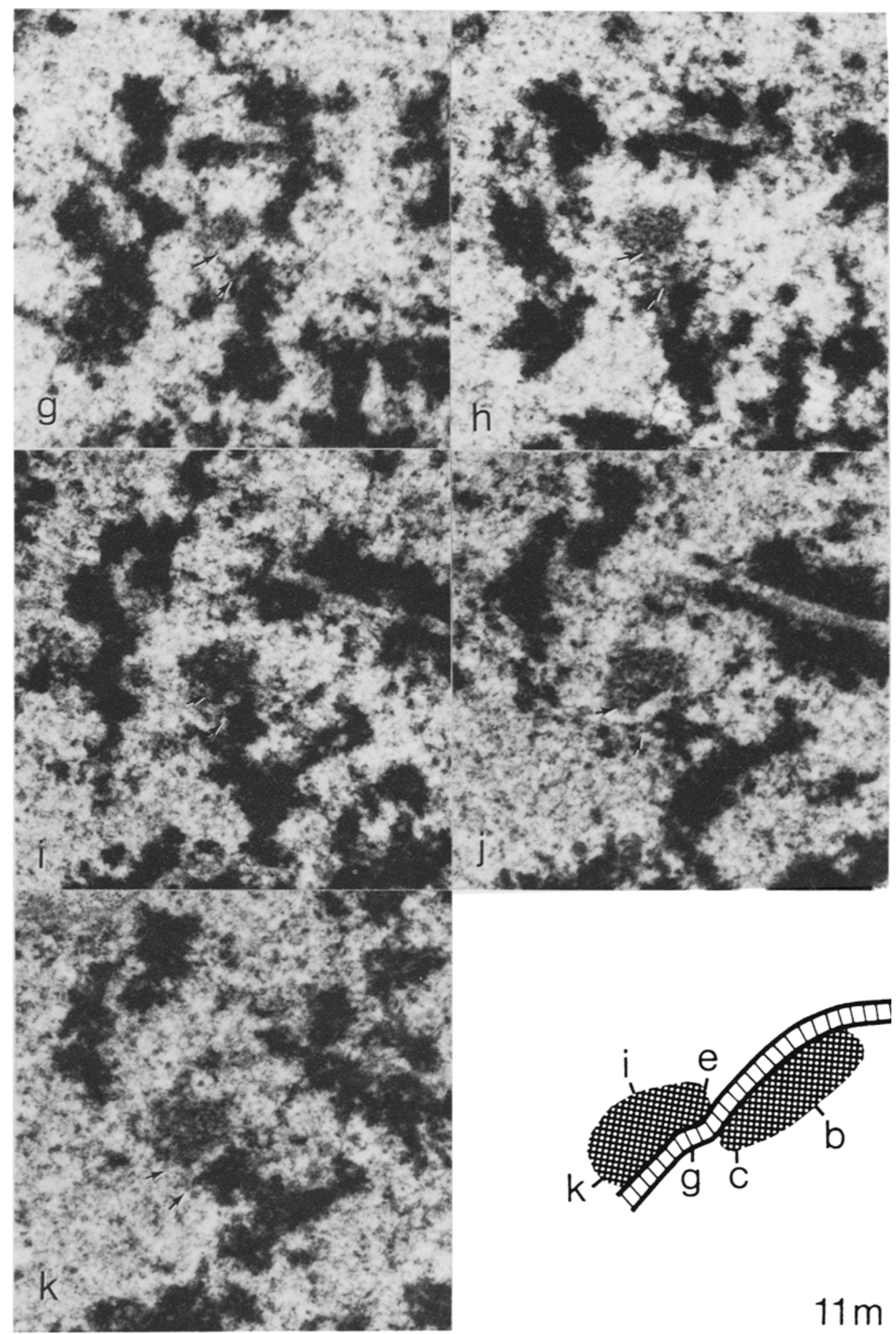


21 was close to the hexavalent shown in Figure 18 and may be part of the broken B chromosomes (at the knob) in the hexavalent. The broken end of chromosomes B in Figure 21 was relatively close to the unattached end of the acentric fragment of the association depicted in Figure 20 indicating that the two fragments belong to the same bivalent. Finally, the centromere region of chromosome $\mathrm{C}$ is associated with only one of the lateral components suggesting that chromosome $\mathrm{C}$ is a univalent rather than a broken chromosome pair. A continuity of the lateral component at the tip of the proposed hairpin could, however, not be demonstrated. The foldback pairing of the univalent has resulted in interlocking of three chromosome pairs (chromosomes B in Figure 16 and chromosomes A in Figures 17 and 19), between the unpaired lateral component segments of the hairpin.

In addition to the major deviations from a regular bivalent formation described above, minor local irregularities were observed in the reconstructed late zygotene nucleus. The breakage of the chromosome pairs has already been described as has the prominent interlocking involving the univalent and the three chromosome pairs. A second interlocking was found in the quadrivalent shown in Figure 15 where chromosomes B are entrapped by a small segment of chromosomes A. The relatively long unpaired segment of the A chromosomes in Figure 20 in which one of the lateral components is broken may be indicative of a previous interlocking.

Yet unpaired small chromosome regions, not due to interlocking, were identified in one of the quadrivalents (chromosomes B in Figure 14), in the hexavalent (chromosomes $\mathrm{A}$ in Figure 18) and in the broken chromosome in Figure 21. Finally, two cases of single lateral component loops, one of them foldback paired with a syn- aptonemal complex, were revealed in otherwise regularly paired chromosome regions (chromosomes $B$ of the quadrivalent in Figure 15 and chromosomes $\mathrm{C}$ of the hexavalent in Figure 18).

The present results unequivocally demonstrate that chromosome pairing and synaptonemal complex formation in hexaploid wheat are not confined to homologous chromosomes. The extensive multivalent formation shows that pairing and synaptonemal complex formation frequently occur also between possibly homoeologous chromosomes. Synaptonemal complex formation in the univalent portions of the pentavalent, the nonhomologously paired centromere regions, the foldback pairing of the univalent and the small interstitial hairpin furthermore show that synaptonemal complexes also form between nonhomologous chromosome regions. In this context it is important to stress that the synaptonemal complex of chromosome regions most likely homologously paired and the complex between obviously nonhomologously paired chromosome regions do not differ in ultrastructure.

\subsection{Pachytene}

\subsubsection{Organization of the pachytene nucleus}

In the completely reconstructed early pachytene nucleus, the polarized distribution of the telomeres was less prominent than at late zygotene, most of the attachment sites being within about one half of the nuclear membrane (Figure 7). Seven of the 42 pairs of telomeres were free in the nucleoplasm, while the rest were attached to the inner membrane of the nuclear envelope.

The total length of the lateral components amounts to $2470 \mu \mathrm{m}$ compared to a value of $3784 \mu \mathrm{m}$ for the late zygotene nucleus. At early pachytene the nucleus is ellipsoid with a mean diameter of $16 \mu \mathrm{m}$. The mean chromosome

Figure 11. Eleven consecutive sections showing pairing and synaptonemal complex formation between centromere $(\mathrm{Ce})$ and noncentromere regions.

A reconstruction of the eleven sections is given in Figure $11 \mathrm{~m}$ and a reconstruction of the entire chromosome pair is shown in Figure 20 (chromosomes A). The center-to-center distance between the two centromere regions is $1.3 \mu \mathrm{m}$. The lateral components are denoted by arrows. $(B a r=0.5 \mu \mathrm{m})$. 


\section{Table I}

Absolute and relative synaptonemal complex (SC) lengths, centromere indices and number of recombination nodules of the 21 pachytene bivalents of Triticum aestivum. The light microscopic data on lengths and centromere indices for telophase II chromosomes of SEARS (35) are included for comparison (LM). The bivalents and chromosomes are arranged according to decreasing length.

\begin{tabular}{|c|c|c|c|c|c|c|}
\hline \multirow{2}{*}{$\begin{array}{l}\text { Bivalent } \\
\text { or } \\
\text { chromosome }\end{array}$} & \multirow{2}{*}{$\begin{array}{l}\text { Absolute } \\
\mathrm{SC} \text { length } \\
\text { in } \mu \mathrm{m}\end{array}$} & \multicolumn{2}{|c|}{ Relative length } & \multicolumn{2}{|c|}{$\begin{array}{l}\text { Centromere } \\
\text { index }\end{array}$} & \multirow{2}{*}{$\begin{array}{c}\text { Number of } \\
\text { recombination } \\
\text { nodules }\end{array}$} \\
\hline & & EM & $\mathrm{LM}$ & EM & $\mathrm{LM}$ & \\
\hline 1 & 100 & 8.2 & 6.9 & 30 & 44 & 7 \\
\hline 2 & 75 & 6.1 & 6.4 & 28 & 27 & 7 \\
\hline 3 & 72 & 5.8 & 6.1 & 45 & 44 & 4 \\
\hline $4 b$ & 70 & 5.7 & 5.9 & 44 & 42 & 2 \\
\hline 5 & 67 & 5.5 & 5.5 & 35 & 36 & 3 \\
\hline $6 \mathrm{~b}$ & 67 & 5.4 & 5.1 & 33 & 48 & 6 \\
\hline $7 a$ & 66 & 5.4 & 5.1 & 49 & 45 & 8 \\
\hline $8 a$ & 64 & 5.2 & 5.1 & 37 & 46 & 6 \\
\hline 9 & 60 & 4.8 & 5.1 & 40 & 47 & 2 \\
\hline 10 & 60 & 4.8 & 4.9 & 42 & 44 & 5 \\
\hline 11 & 58 & 4.7 & 4.8 & 43 & 46 & 3 \\
\hline 12 & 57 & 4.6 & 4.6 & 45 & 45 & 4 \\
\hline 13 & 56 & 4.5 & 4.6 & 46 & 44 & 3 \\
\hline 14 & 55 & 4.4 & 4.4 & 49 & 39 & 0 \\
\hline 15 & 54 & 4.4 & 4.2 & 37 & 42 & 6 \\
\hline $16 \mathrm{~b}$ & 49 & 4.0 & 4.2 & 47 & 34 & 8 \\
\hline 17 & 47 & 3.8 & 3.9 & 28 & 36 & 1 \\
\hline 18 & 46 & 3.7 & 3.5 & 35 & 47 & 2 \\
\hline 19 & 41 & 3.3 & 3.3 & 36 & 47 & 4 \\
\hline $20 \mathrm{~b}$ & 40 & 3.2 & 3.2 & 18 & 36 & 6 \\
\hline 21 & 31 & 2.5 & 3.1 & 39 & 35 & 1 \\
\hline Mean & 1,235 & & & & & 88 \\
\hline
\end{tabular}

a) Nucleolus organizing region identified in the pachytene nucleus.

b) Chromosomes with secondary constrictions at metaphase in root tip cells (4).

length is $59 \mu \mathrm{m}$ which is 3.7 times the mean nuclear diameter, the corresponding value for late zygotene being 5.3 times.

A drastic reduction in the number of irregularities in chromosome pairing has occurred between late zygotene and early pachytene: All 42 chromosomes were paired into bivalents at early pachytene, each with a continuous synaptonemal complex from telomere to telomere as shown in Figures 22 to 28 . In addition, two acentric fragments were present (Figures 27 and 28). Pairing and synaptonemal complex formation appear at this stage to be exclusively between homologous chromosomes as judged by the identical lengths of the two lateral components of each bivalent, and the apparently homologous 
pairing of all centromere regions. Since the free ends of the broken bivalents cannot be distinguished from free telomeres, it is not possible to determine to which of the bivalents with a free end the fragments belong. It is, however, likely that one fragment belongs to one of the nucleolus organizing bivalents, as both bivalents 8 (Figure 22) and 7 (Figure 27) are discontinuous inside the nucleolus, but only one acentric bivalent segment emerges from the nucleolus. Hence, one of these bivalents must be broken near the nucleolus organizing region. In order to provide the best fit with the light microscopic data on chromosome length and centromere indices, one of the acentric fragments has been assigned to bivaient 7 (Figure 27). The other segment has more arbitrarily been assigned to the free end of bivalent 6 (Figure 28) in order to improve the agreement in length with the light microscopic karyotype (see section 3.4.2.).

It is thus evident that the pairing pattern observed at late zygotene has undergone a remarkable modification whereby multivalents as well as associations of more than two chromosomes have been transformed into homologously paired bivalents. Local irregularities in the form of interlockings and interstitial hairpins were not identified, and only two cases of discontinuous synaptonemal complexes were observed compared to four cases of discontinuous lateral components and two cases of discontinuous synaptonemal complexes present at late zygotene. Hence, the correction of the zygotene chromosome pairing in wheat appears to include also the resolution of interlockings as well as repair and reunion of chromosome breaks.

\subsubsection{The pachytene karyotype}

The absolute length, relative length and centromere index of the 21 pachytene bivalents are presented in Table $I$ and in the idiogram in Figure 29. The data reveal a gradual decrease in length from the longest to the shortest bivalent, the range being from 100 to $31 \mu \mathrm{m}$. The corresponding relative lengths decrease from 8.2 to 2.5 percent. A similar continuous spectrum is also evident for the centromere indices ranging from a maximum of 49 to a minimum of 18 percent.

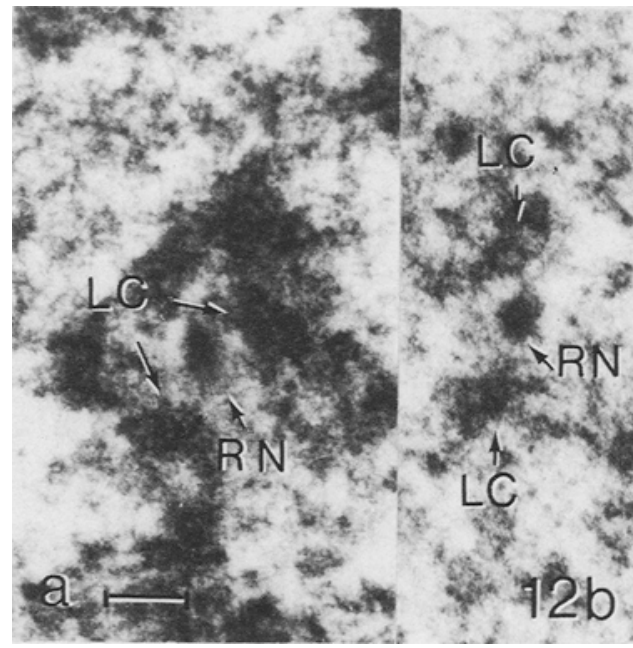

Figure 12. High magnifications of an oblique section (a) and a cross section (b) of synaptonemal complexes with recombination nodules (RN).

The nodules are associated with the central region of the synaptonemal complex. LC, lateral component. $(\operatorname{Bar}=0.2 \mu \mathrm{m})$.

These results are basically similar to the light microscopic data by SEars (35) who measured length and arm ratio of the univalent at telophase II in lines monosomic for each of the 21 different chromosomes. As seen in Table I, the range of the relative length is from 6.9 to 3.1 percent and from 49 to 28 percent for the centromere indices with a nearly continuous spectrum of values between the minimum and the maximum.

It is thus not possible to identify the individual chromosomes or even to allocate them to major groups on the basis of lengths and centromere indices and a detailed comparison between the present data and the light microscopic data is only possible for the nucleolus organizing chromosomes.

Light microscopic and biochemical analyses have revealed four chromosomes capable of organizing a nucleolus $(4,7,12)$. Two organizer regions were found in the reconstructed pachytene nücleus and all four in the late zygotene nucleus. The relative length of the synaptonemal complex and the centromere index for the two organizer bivalents at pachytene are very similar to the relative length and centromere index of the two longest of the four organizer chromosomes at telophase II (Table I) in agreement with the 
observation from other studies that the meiotic karyotype established by electron microscopy agrees well with the mitotic and meiotic karyotypes established by light microscopy ( 13 , 16).

\subsection{Recombination nodules}

Electron dense recombination nodules were identified in the central region of the synaptonemal complex at mid and late zygotene as well as at early pachytene (Figure 12). The recombination nodules vary in shape from spheres to ellipsoids, the shorter diameter ranging from 30 to $80 \mathrm{~nm}$ and the longer from 30 to $130 \mathrm{~nm}$. Often, the nodules appear to be connected to both lateral components by fine filaments (Figure 12).

The total number of recombination nodules in the late zygotene nucleus amounts to 97, apparently distributed at random among the synaptonemal complexes of the nucleus. The five bivalents (Figure 13) contain from 0 to 7 nodules, the four quadrivalents (Figures 14-17) from 5 to 13 , while 19 nodules were present in the hexavalent (Figure 18). The two fragments depicted in Figure 19 had 1 and 2 nodules, the foldback paired univalent (Figure 21) had 4 nodules, the association of two chromosomes and two fragments (Figure 20) had 5 and the association of five chromosomes shown in Figure 19 had 16 recombination nodules of which 3 were located in the synaptonemal complex combining fragments constituting the fifth chromosome.

As pointed out previously, it is not possible to establish unequivocally whether pairing and synaptonemal complex formation at a given site in the multivalents and in the multiple associations are between homologous chromosome segments. It is, however, reasonable to conclude that the foldback pairing of the univalent as well as the synaptonemal complex between the fragments in the association of five chromosomes are between nonhomologous chromosome segments. If this is the case, then 7 recombination nodules are present in the nonhomologously paired regions.

At early pachytene, the total number of recombination nodules is 88 and as in late zygotene, these are apparently distributed randomly among and along the 21 bivalents and the two bivalent fragments. The number of recombi- nation nodules per bivalent is given in Table I and their position is shown in the idiogram (Figure 29). The number of recombination nodules per bivalent ranges from 0 in bivalent 14 , to 8 in bivalents 7 and 16 .

\section{DISCUSSION}

\subsection{General aspects of chromosome pairing}

Recent observations in a number of organisms have demonstrated that the presence of perfectly paired homologous chromosomes at the pachytene stage of meiosis does not reflect an equally perfect synapsis of the homologues during the initial pairing and synaptonemal complex formation at zygotene (see 28 for review).

Crossing over and chiasma formation are absent in the female sex of the silkworm, Bombyx mori, and the chromosome pairing can therefore be analyzed without being affected by the exchange of nonsister chromatids. In tetraploid oocytes, homologously paired quadrivalents are invariably formed during the initial synapsis at zygotene whereas at mid pachytene, only bivalents are present (27). This shows that, in the absence of crossing over, chromosome pairing is a dynamic process capable of transforming multivalents into bivalents. As judged by the low frequence of univalents, the dissolution of the central region of the synaptonemal complex distal to the shift of pairing partners is immediately followed by the formation of a new central region between a different combination of lateral components (27). The bivalents are maintained up to metaphase $\mathrm{I}$, the disjunction is regular and tetraploid Bombyx females are fully fertile (1). In contrast, tetraploid Bombyx males are sterile and contain in their metaphase I plates approximately the same number of multivalents as the late zygotene nuclei of tetraploid oocytes (19). This unambiguously demonstrates that the occurrence of crossing over effectively prevents the transformation of multivalents into bivalents.

The analysis of chromosome pairing in triploid Bombyx oocytes (25) has furthermore revealed that the initially formed trivalents are subjected to a similar correction yielding bivalents and univalents but, in the absence of the potential for complete homologous synapsis, the left-over univalents undergo a nonhomologous synapsis and synaptonemal complex formation 
giving rise to associations of two or more chromosomes and foldback paired univalents. In the absence of homology, the turnover of the synaptonemal complexes during the correction process may thus lead to pairing also between nonhomologous chromosomes and chromosome segments.

Complete reconstructions from serial sections have finally revealed that nearly all the analyzed late zygotene Bombyx oocytes (24), human spermatocytes (26) and Bombyx spermatocytes (17) contained one or more interlocking. Concomitantly, chromosome and bivalent breaks were frequently observed, often in regions of interlockings or presumptive interlockings. By early pachytene, virtually all interlockings and breaks have disappeared both in Bombyx oocytes and in human and Bombyx spermatocytes $(17,24,26$, 29). This shows that interlockings are regularly formed during the zygotene pairing but again resolved before early pachytene and that the mechanism responsible for their resolution includes a transient breakage of chromosomes or bivalents.

Hence, the zygotene pairing is not faultless either in diploids or in poiypioids but is subsequently modified through the action of mechanisms which are capable of optimizing pairing in the form of free bivalents. The occurrence of crossing over effectively terminates correction processes which involve dissolution and reassembly of synaptonemal complexes.

\subsection{Chromosome pairing in wheat}

The most significant observation in the present study is that the highly irregular zygotene pairing with extensive multivalent formation, nonhomologously paired centromere regions, small interstitial loops of unpaired lateral components and interlockings undergoes a remarkable correction resulting at early pachytene in pairing exclusively in the form of bivalents. This observation is in full agreement with the general scheme for chromosome pairing described in the previous section and definitely invalidates the hypothesis of an alignment of homologous chromosomes prior to the meiotic prophase as the primary cause of exclusive bivalent formation at pachytene and later stages in allohexaploid wheat. A detailed interpretation of the pairing pattern of the analyzed late zygotene nucleus with respect to homology and homoeology of the chromosomes involved in the multiple associations is not possible on the basis of absolute criteria. It is, however, reasonable to assume that the vast majority of the zygotene pairing in wheat is between homologous chromosomes and chromosome segments as is the case in all other organisms investigated by three dimensional reconstructions. Hence, the multiple associations have originated by shifts of pairing partners between homoeologous chromosomes. The observation that shifts of pairing partners very frequently are present in adjacent pairs furthermore shows that pairing of homoeologous chromosomes with few exceptions is initiated interstitially while pairing between the homologues apparently has occurred preferentially in telomere regions.

Although pairing and synaptonemal complex formation of the relatively long wheat chromosomes are expected to be initiated in several interstitial regions as is the case in for example Lilium (15) and Zea (14), telomeric intiation is facilitated both by the attachment of the telomeres to the nuclear envelope whereby telomere movements are restricted to a two dimensional space and by the aggregation of the attachment sites. In several organisms, initiation of pairing is almost exclusively telomeric but also in maize, the primary initiation of pairing is very frequently at the telomere regions while the interstitial initiation sites are secondary (5). Hence, it is conceivable that the regular bivalent formation in allohexaploid wheat to some extent relies upon preferential recognition of homologous telomere regions. In addition, it is to be expected that the extent of pairing between homologues also when initiated interstitially will exceed pairing between the homoeologues and thus stimulate correction towards homologous bivalents.

The pairing pattern of the association of five and in the univalent is, however, not explicable in terms of initiation of pairing exclusively between homologous telomere regions. At least in some of the telomere regions pairing is obviously between homoeologous chromosomes and in the univalent (Figure 21, C) possibly between nonhomologous regions. These chromosome associations may reflect an initial 


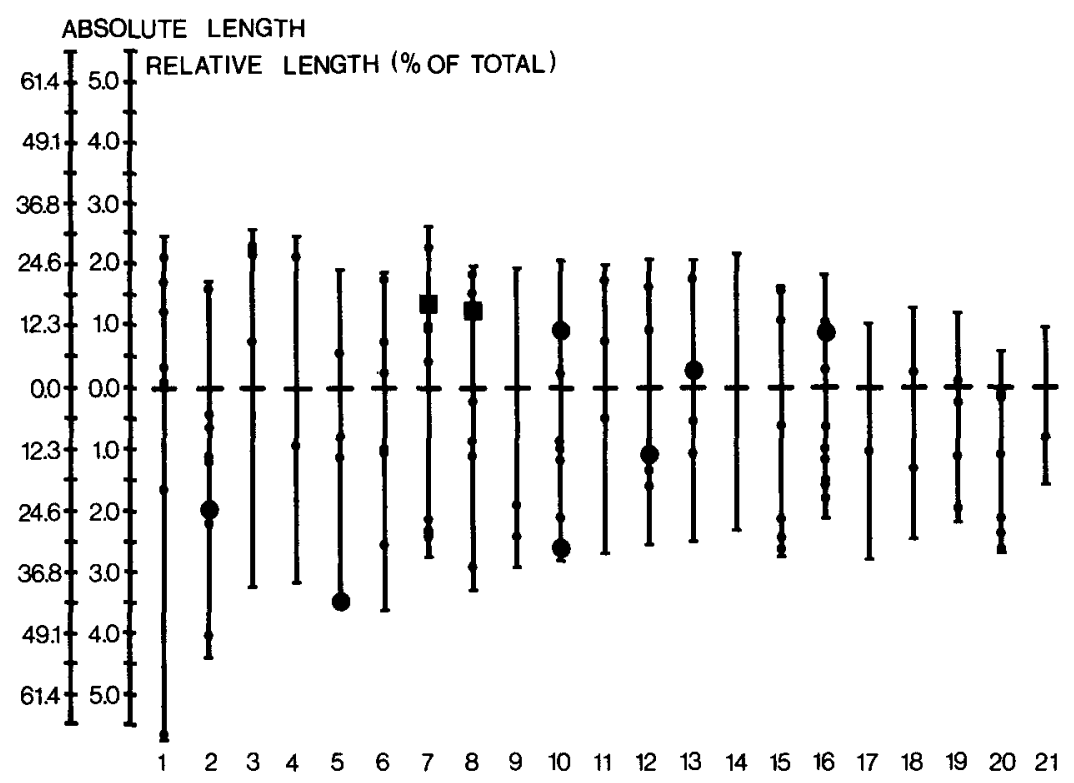

Figure 29. Idiogram showing the absolute and the relative synaptonemal complex length of the 21 pachytene bivalents.

The squares denote the position of the nucleolus organizing regions, the large filled circles the position of dense knobs and the small filled circles the position of recombination nodules.

failure of homologous synapsis of the telomere regions followed by apparently homologous synapsis of the nontelomeric regions (bivalents $\mathrm{A}$ and $B$ and univalent $C$ in Figure 19) and nonhomologous synapsis and synaptonemal complex formation between the fragments of this univalent. Hence, the correction of pairing towards homologous bivalents may in this case proceed to the telomere regions. Whether or not the correction mechanism is capable of transforming also the fifth chromosome (the $\mathrm{C}$ fragments) and the univalent into a homologously paired bivalent is uncertain. It should be noted that univalents are not uncommon at metaphase I in normal hexaploid wheat (32).

The complexity of these associations is not exceptional to wheat. Also in normal diploid organisms such as Lilium (15), humans (26) and Bombyx $(17,29)$, the pairing pattern at late zygotene often appears very complex with clear cases of small nonhomologously paired chromosome segments, regions of multiple interlockings and frequent chromosome and bivalent breakage. These irregularities are nearly all corrected/ repaired by early pachytene.
The smaller local irregularities within otherwise regularly paired chromosome regions in the analyzed late zygotene nucleus may be indicative of minor chromosomal rearrangements. The nonhomologously paired centromere regions were found in both late zygotene nuclei and may reflect heterozygosity for a pericentric inversion. If this is the case, it implies that the correction mechanism is capable of aligning the two centromere regions by their gross structure irrespective of their opposite polarity, as all centromere regions were paired at early pachytene. In agreement with this, recent analyses have shown that in a duplication heterozygote of mouse the correction of pairing may also involve an adjustment of the lateral component length, as the loop formed during the initial pairing is eliminated during pachytene, the heteromorphic bivalent then appearing normally paired throughout its entire length (23).

The presence of two interlockings, one being a simple bivalent interlocking, the other a multiple interlocking involving a univalent and three bivalents are not unexpected considering the very long chromosomes and the presence of several 
initiation sites for synaptonemal complex formation per bivalent. Together with the absence of interlockings and chromosome breaks at early pachytene, the chromosome and bivalent breaks at zygotene are consistent with the mechanism for resolution of interlockings revealed in humans (26), Bombyx spermatocytes (17) and oocytes (29) and provide additional evidence in support of the universality of the break-reunionrepair mechanism, for resolution of interlockings.

By analogy with the situation in tetraploid Bombyx, the present observations furthermore show that in wheat, crossing over between homoeologous chromosomes does not occur before the correction of the zygotene chromosome pairing is completed. Hence, the presence of recombination nodules between apparently homoeologous chromosomes as well as between nonhomologous chromosomes clearly demonstrates that recombination nodules are prerequisites for, rather than the result of, crossing over in agreement with previous investigations (6).

The present results are especially interesting in the light of the investigation by HotтA et al. (18) in the achiasmatic Lilium hybrid Black Beauty: In this hybrid, the meiocytes do not undergo DNA nicking at pachytene despite an apparently normal level of the required specific endonuclease activity. Furthermore, the level of the $\mathbf{R}$ protein activity, a meiosis specific protein facilitating the renaturation of single stranded DNA, was reduced to about one fifth of the level in chiasmatic hybrids. After chromosome doubling induced by in situ colchicine treatment, both the DNA nicking and the normal level of the R protein activity are restored. These experiments demonstrate that incomplete pairing in the hybrid acts as an inhibitor of meiosis specific activities believed to be involved in crossing over. By providing for complete homologous pairing, these activities are restored and crossing over and chiasma formation occur.

Likewise, the determining factor for the onset of crossing over in allohexaploid wheat appears to be the completion of the pairing correction at early pachytene. The gene(s) on the long arm of chromosome 5B may thus control the time of crossing over in relation to the correction of the zygotene chromosome pairing: In the absence of $5 \mathrm{BL}$, the time of crossing over is shifted to mid- late zygotene when correction of multivalent pairing is not yet completed, and multivalents persist to metaphase $I$ as a consequence of chiasma formation between homoeologous chromosomes. Two doses of 5BL postpone crossing over to early pachytene when correction into free bivalents is completed. Six doses of 5BL may delay crossing over so long that optimal conditions for its occurrence have passed resulting in the observed reduction of chiasma frequencies. Alone or in combination with other genes, the Ph gene(s) could be involved in the synthesis, assembly, stabilization or degradation of meiosis specific proteins such as the endonuclease or the $\mathrm{R}$ protein.

In the light of the present findings, it is conceivable that multivalent formation during zygotene is a regular feature of meiosis in polyploid organisms displaying only bivalents at metaphase I. Restriction of crossing over to a period after the correction of pairing can account for disomic inheritance and exclusive presence of bivalents at metaphase I observed in allopolyploid and autopolyploid plant species. In several plant genera (e. g. Avena, Festuca, Gossypium and Nicotiana) the genetic regulation of bivalent formation shows similar features to that reported for wheat (32).

\section{ACKNOWLEDGEMENTS}

I wish to express my sincere gratitude to Professor Diter von Wettstein, Dr. Søren W. Rasmussen and Dr. Preben B. Holm, who besides suggesting the investigation also greatly influenced this work through inspiring guidance and discussions. Their assistance in preparing this communication is also highly appreciated. I especially thank JEAN SAGE for outstanding technical assistance and Dr. JøRGEN LARSEN for providing the plants and for many valuable discussions. Finally, the assistance of BENT Hansen, Børge Petersen, Nina Rasmussen, Inge Sommer, Bibl Stampe Andersen and AnNSofl SteinHoltz is gratefully acknowledged. This work was financially supported by grant 202-76-1 BIO DK and BIO-E-4 I 7-DK(G) from the Commission of the European Communities to Professor D. von Wettstein. 

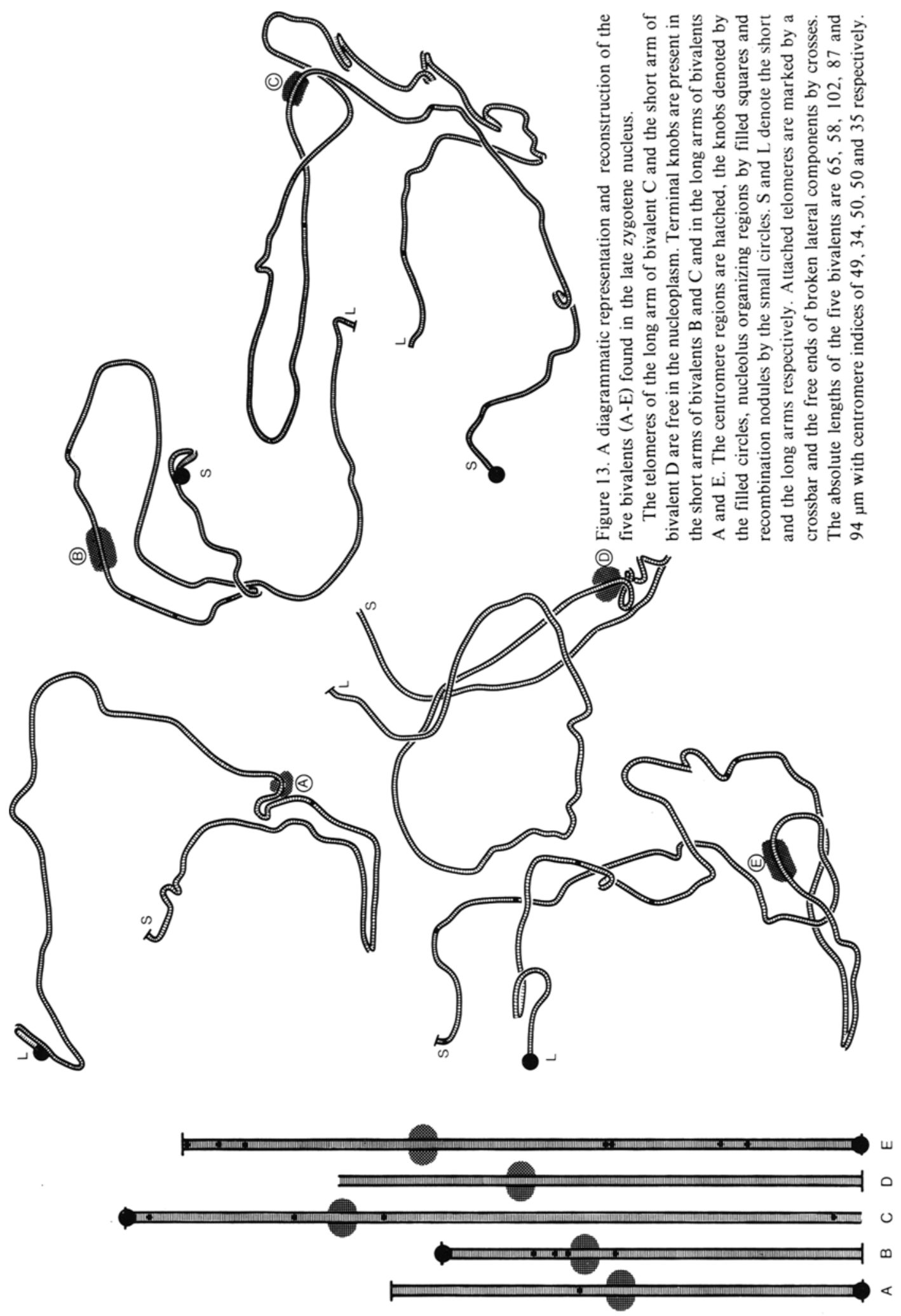

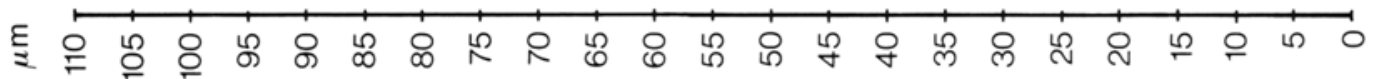



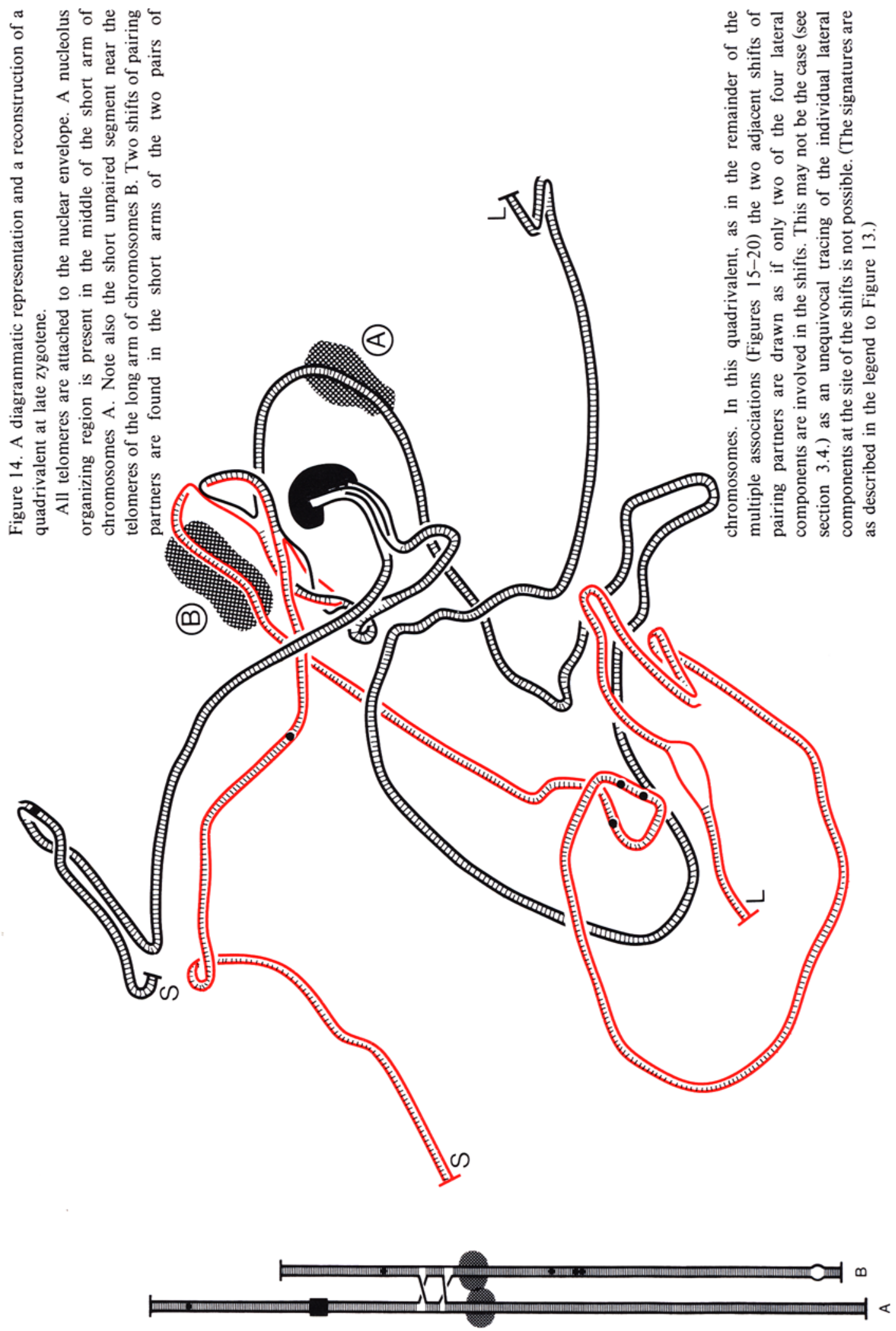

$156 \xi$ 을 잉 \& 

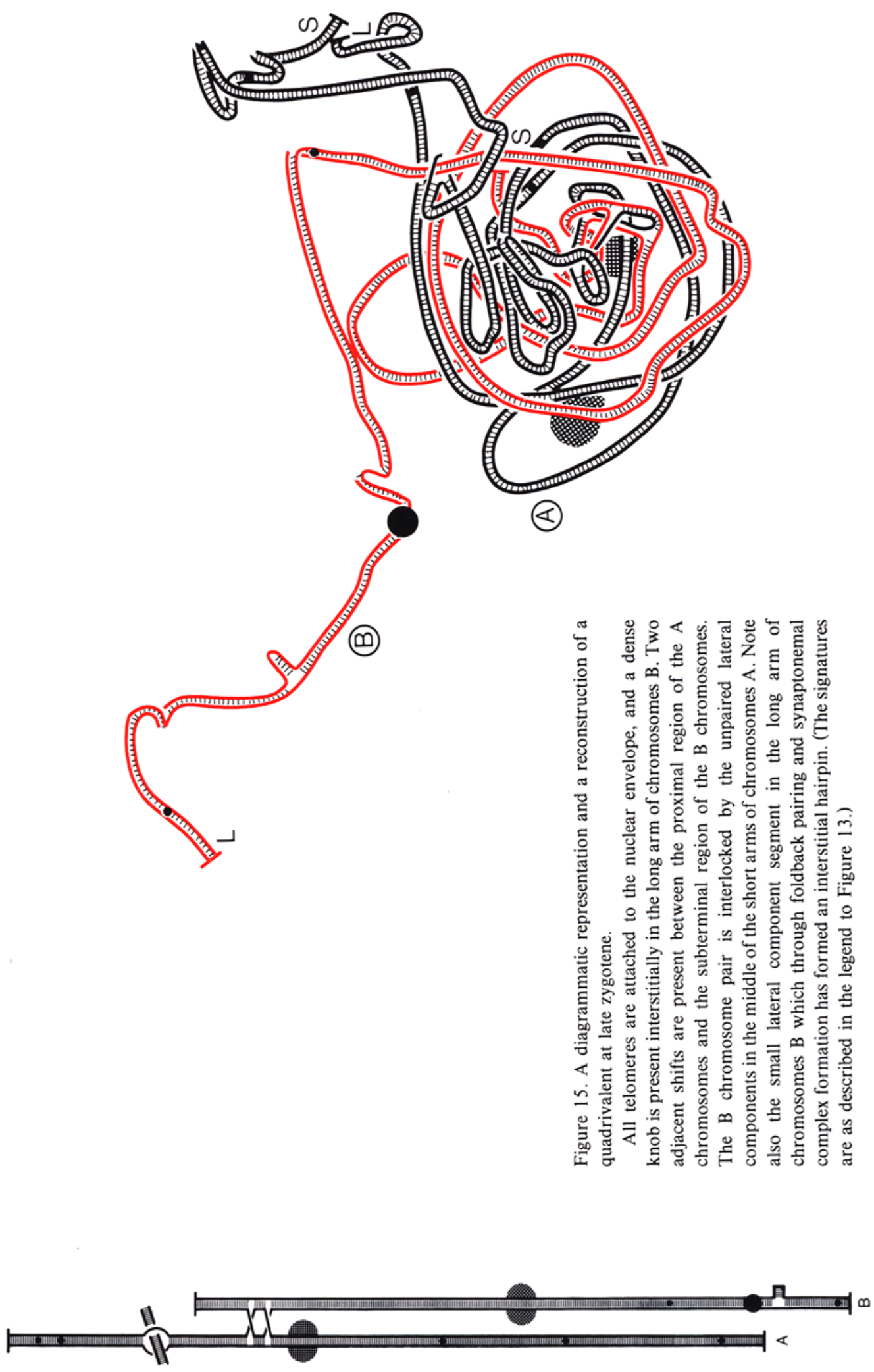

$\xi$ 

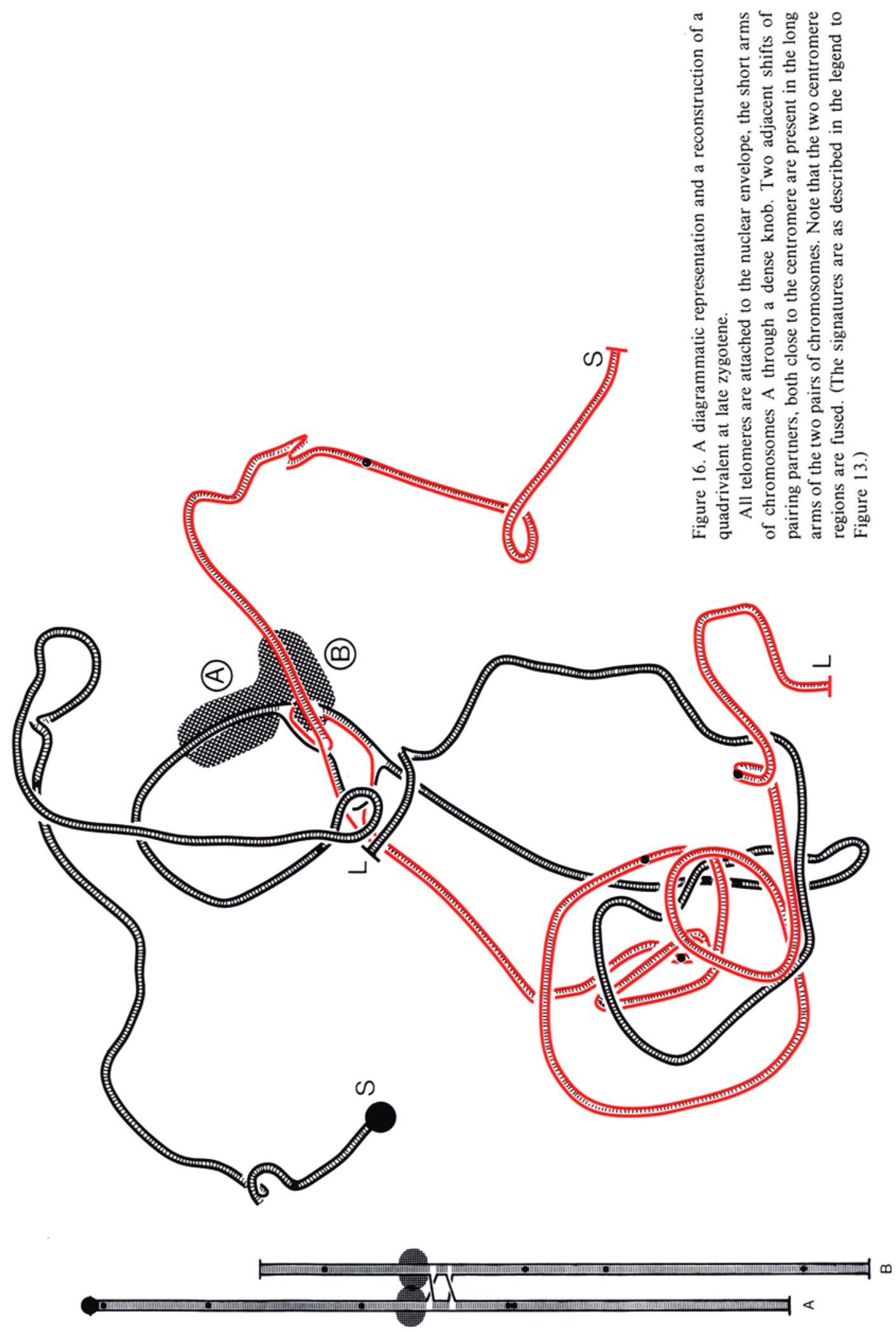

$158 \xi$ 을 으 ம 


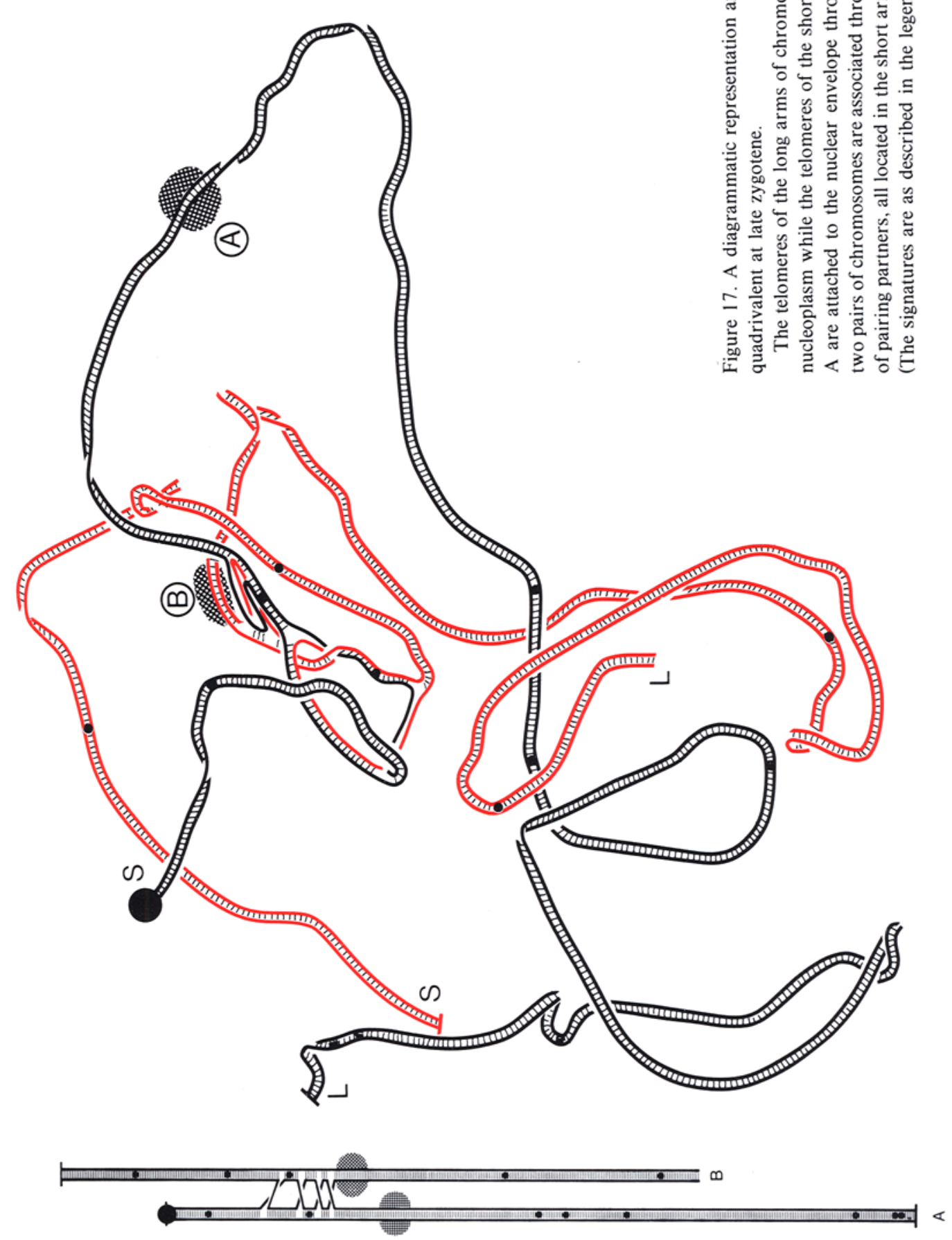

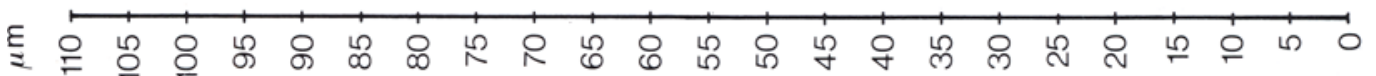




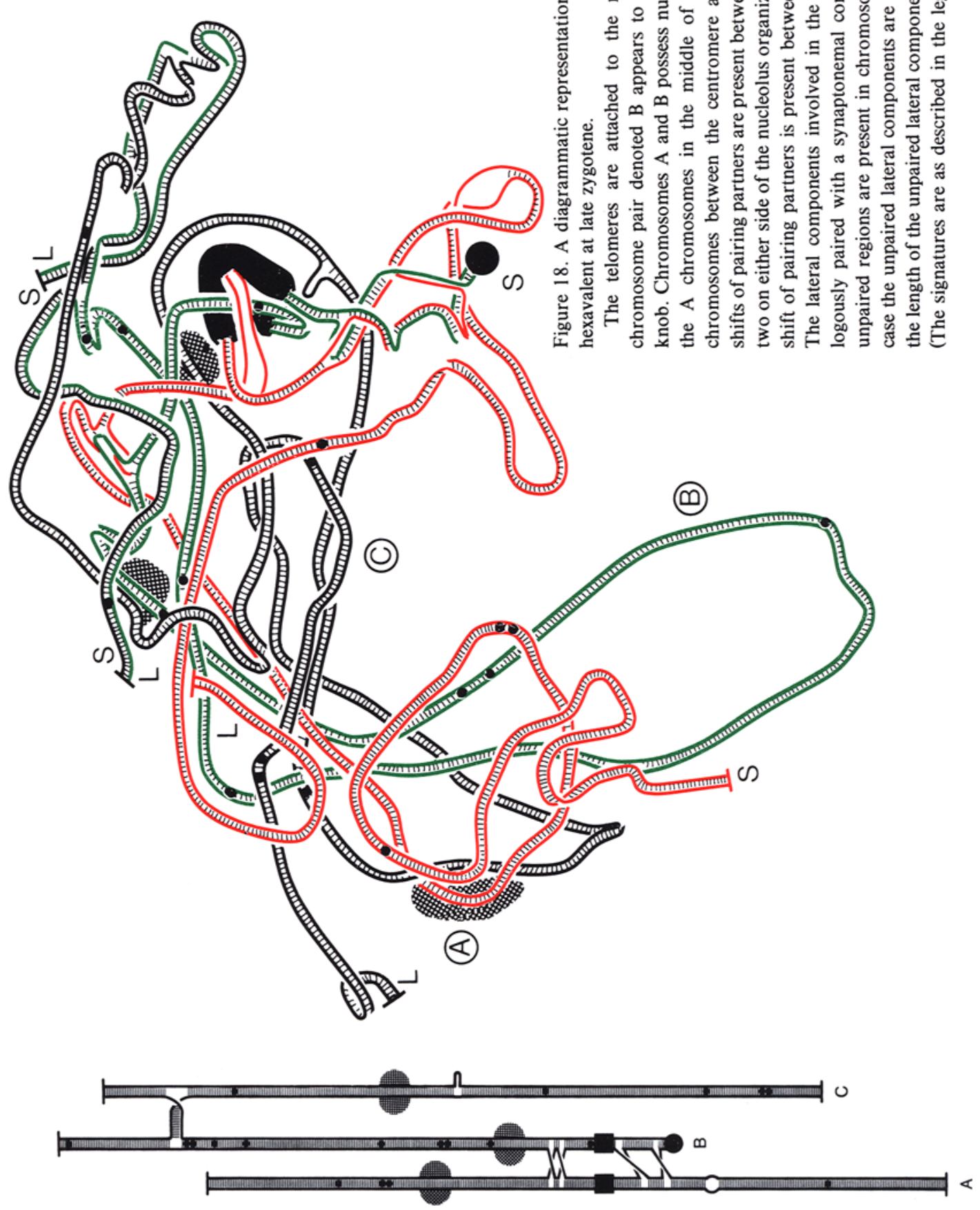

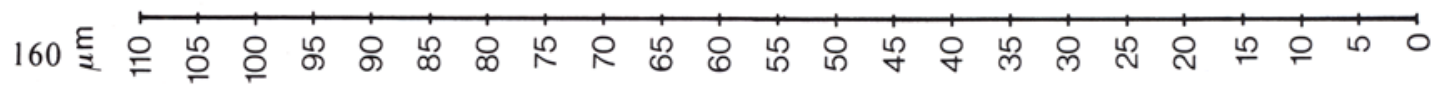




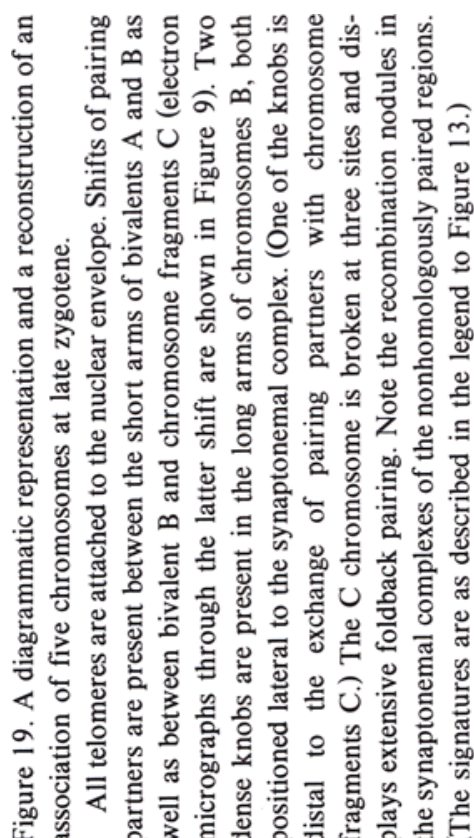
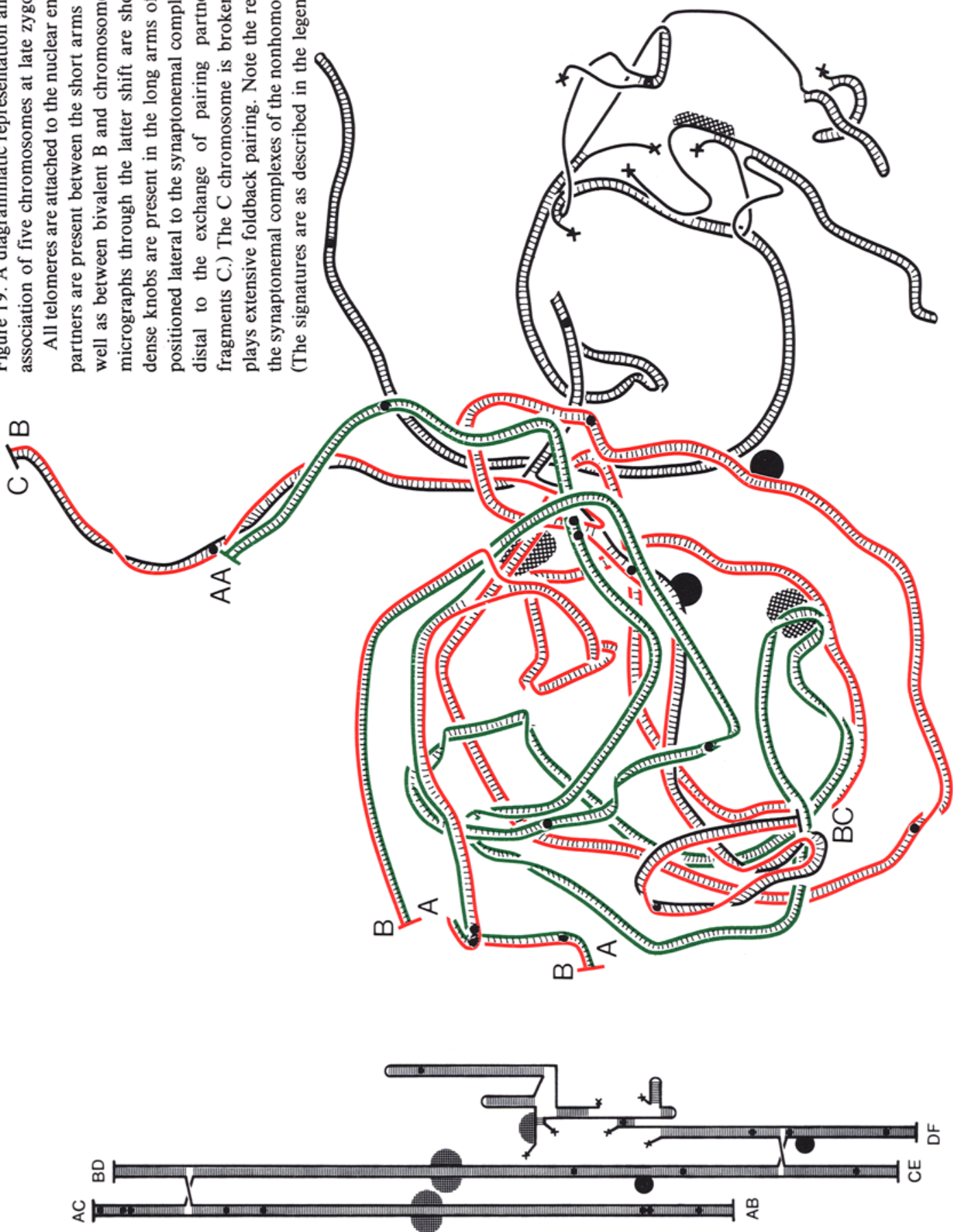

$\xi$ 을 으 으 

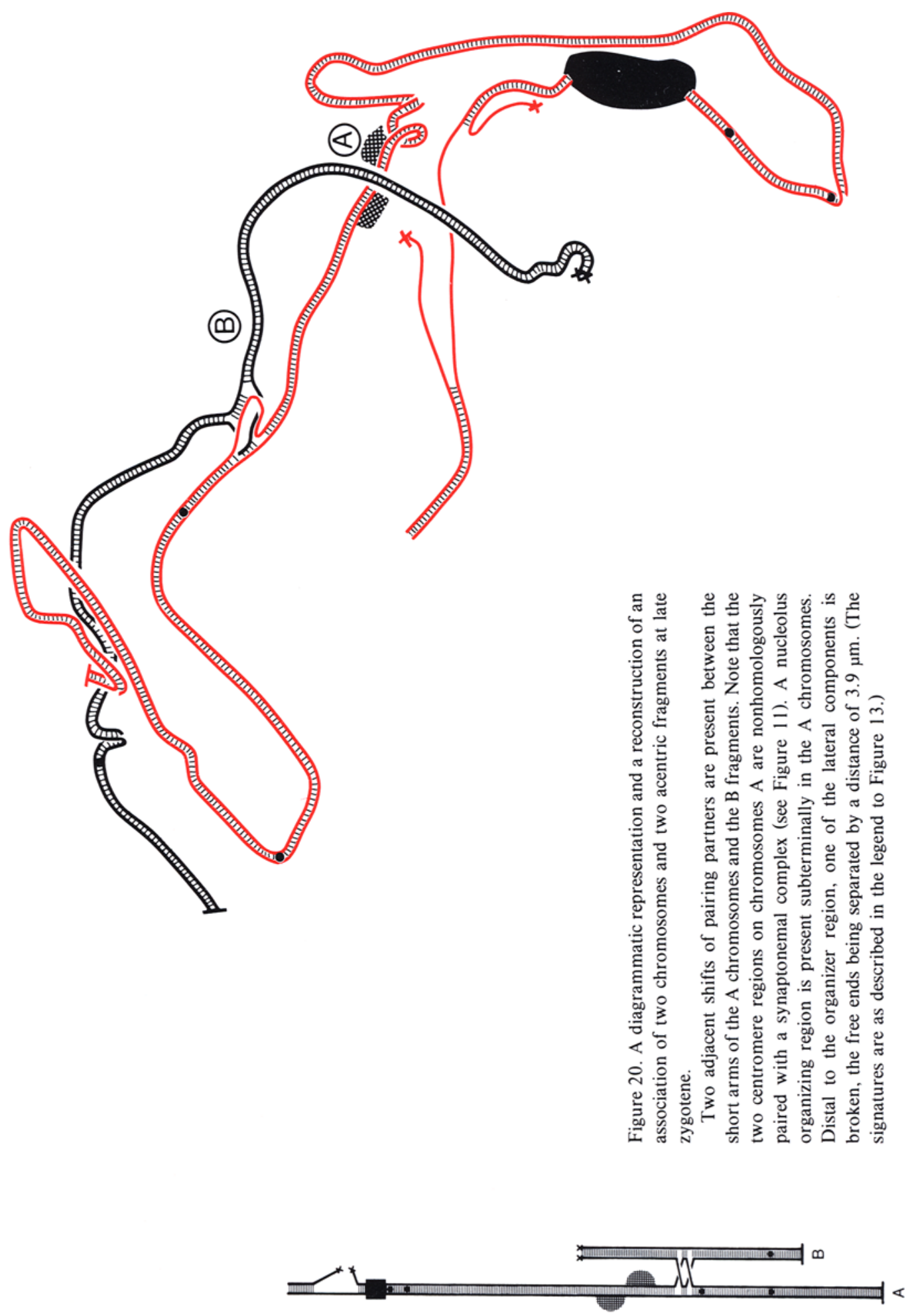

$162 \xi$ 


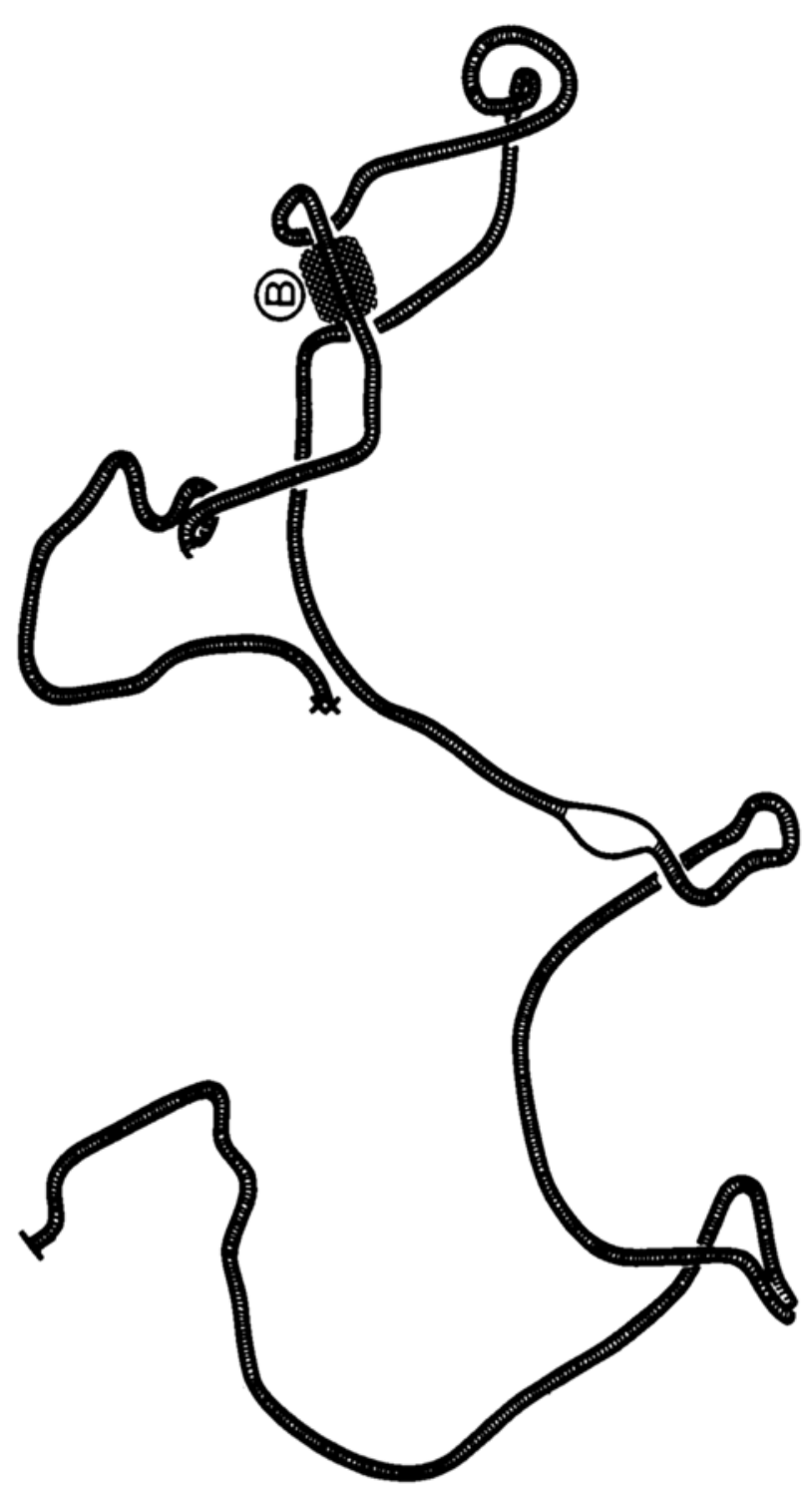

㐫
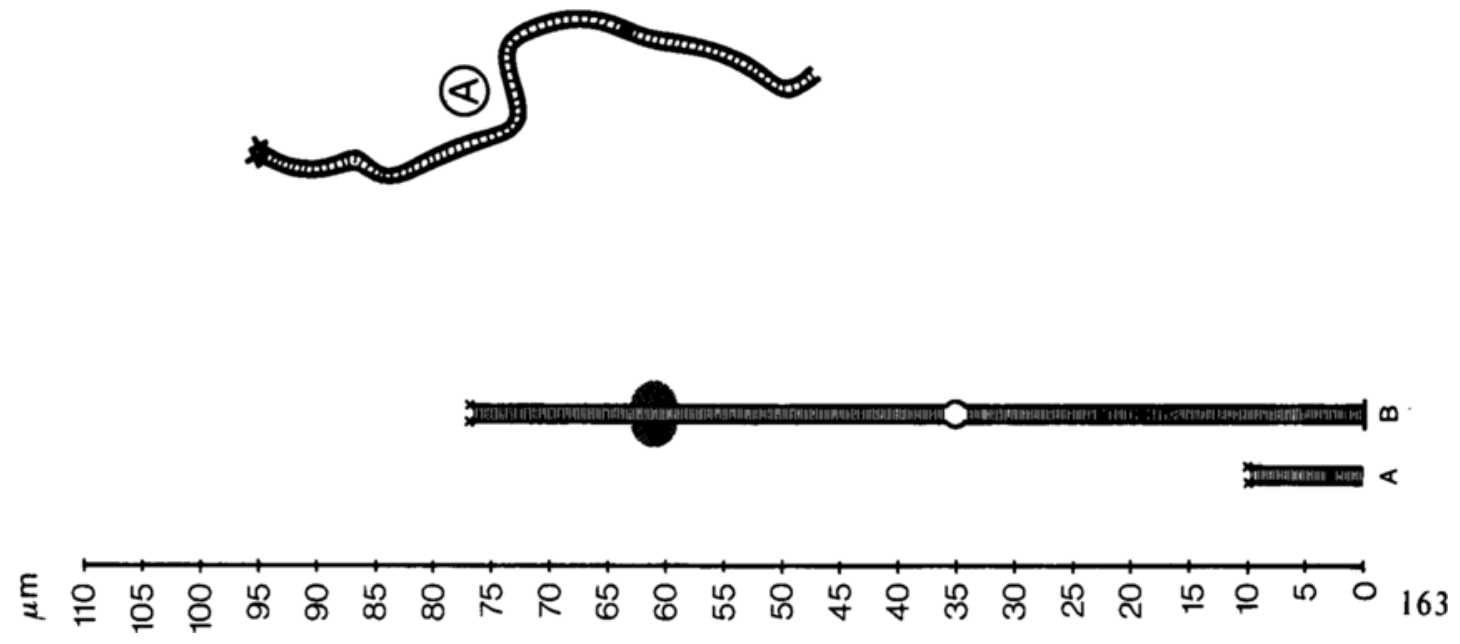

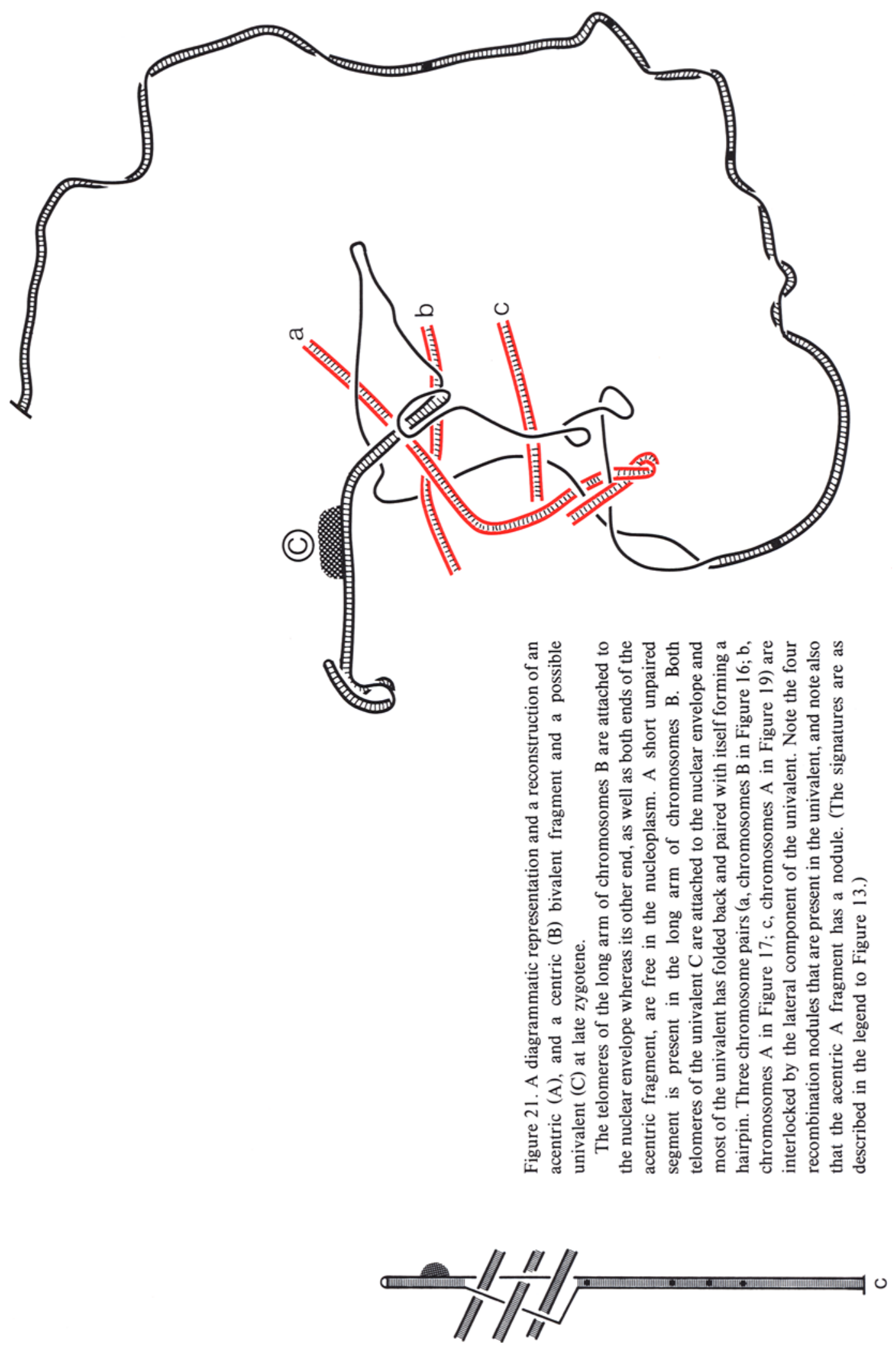

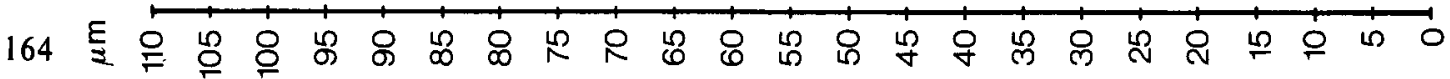




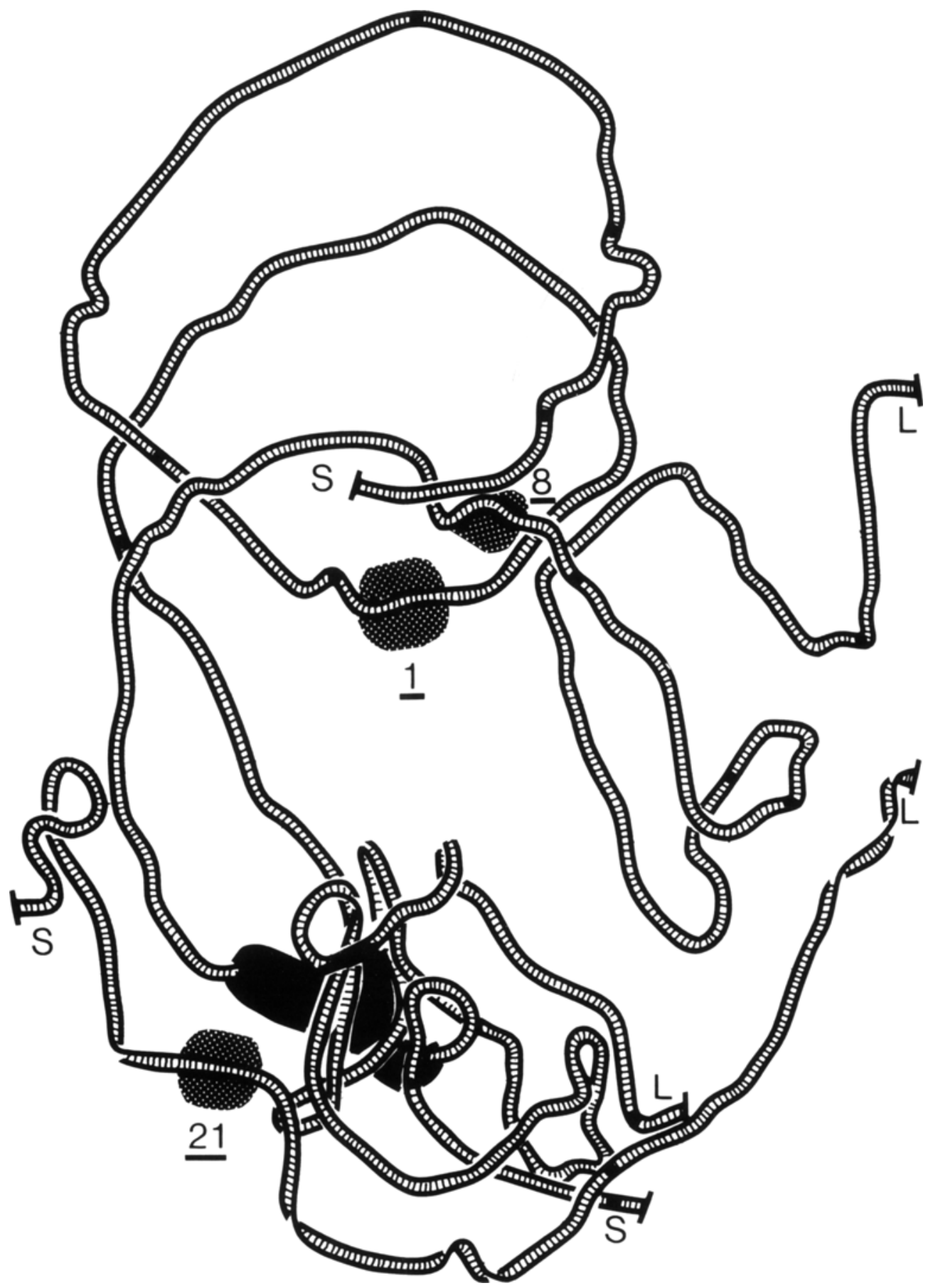

Figure 22. A reconstruction of bivalents 1,8 and 21 at pachytene.

All telomeres are attached to the nuclear envelope. Nucleolar organizing regions are present on bivalents 1 and 8. The organizer region on bivalent 8 is embedded in the nucleolus, and the synaptonemal complex cannot be identified inside the nucleolus. The centromeres of bivalents 8 and 3 (Figure 24) are fused. (The signatures are as described in the legend to Figure 13.) 


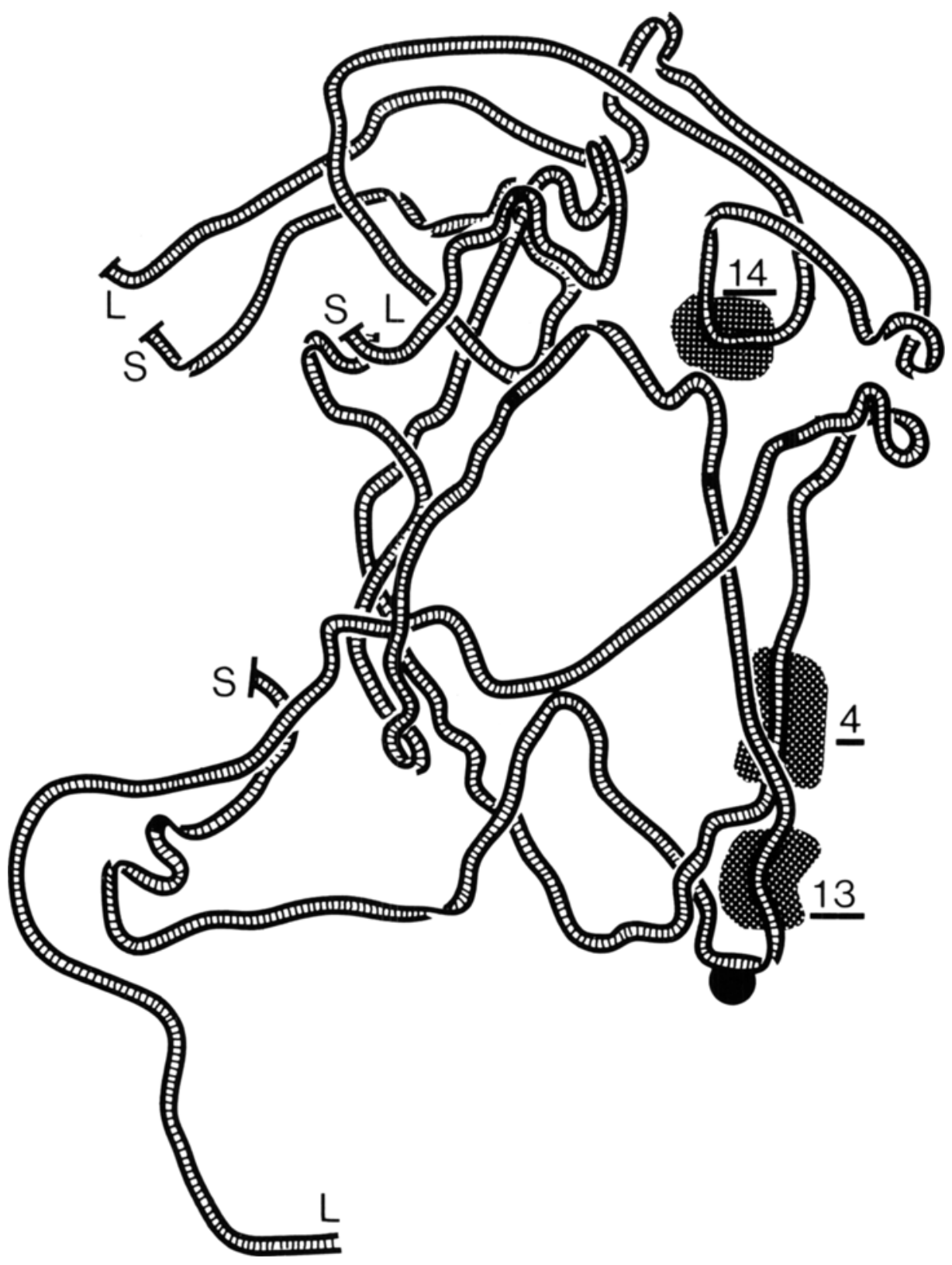

Figure 23. A reconstruction of bivalents 4,13 and 14 at pachytene.

The telomeres of the long arm of bivalents 4 and 13 are free in the nucleoplasm. A dense knob is present close to the centromere on the short arm of bivalent 13. The centromeres of bivalents 13 and 11 (Figure 28) are fused. (The signatures are as described in the legend to Figure 13.) 


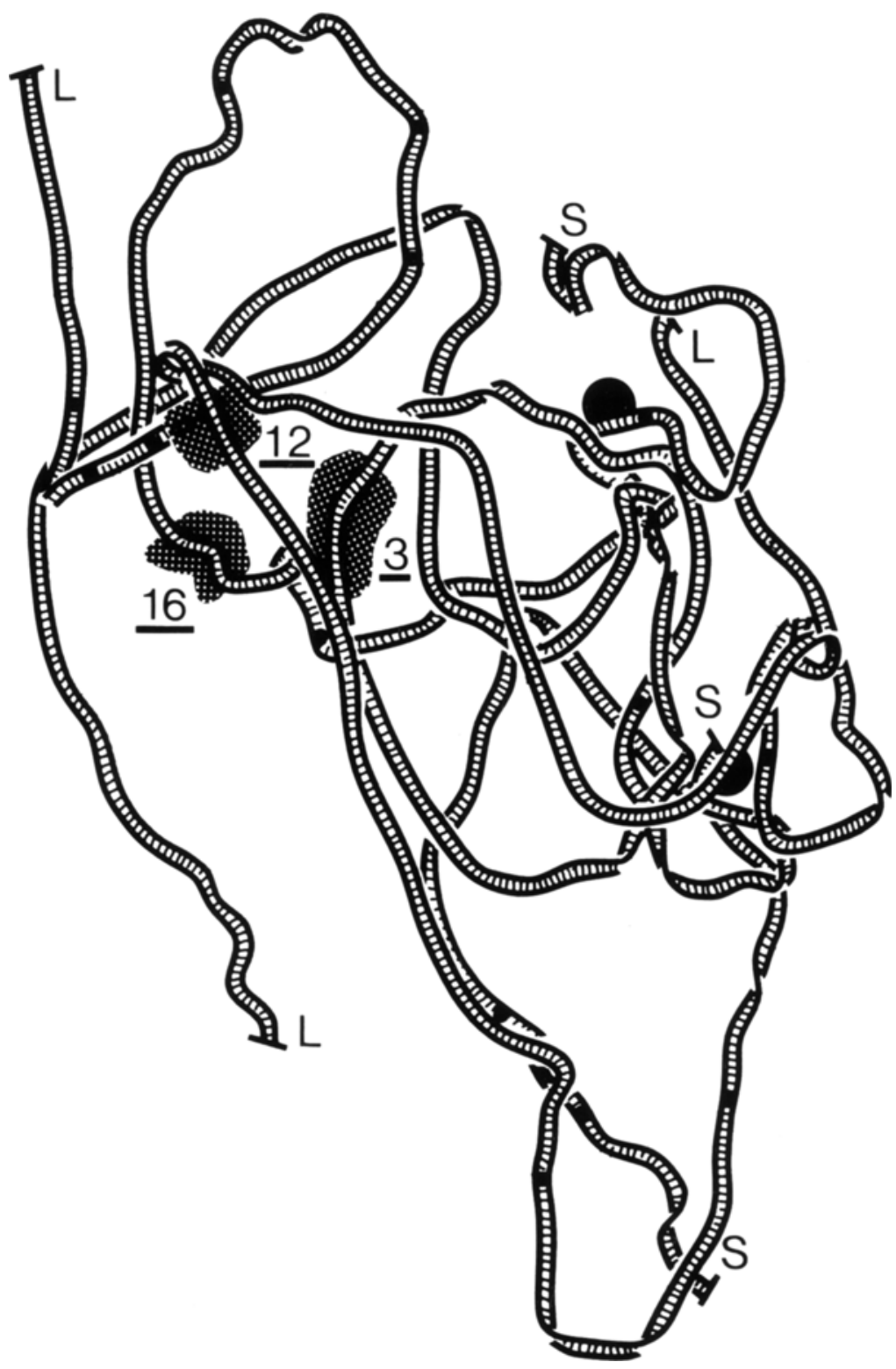

Figure 24. A reconstruction of bivalents 3, 12 and 16 at pachytene.

All telomeres are attached to the nuclear envelope. One knob is present in the long arm of bivalent 12 and one in the short arm of bivalent 16. The centromeres of bivalents 3 and 8 (Figure 22) are fused. (The signatures are as described in the legend to Figure 13.) 


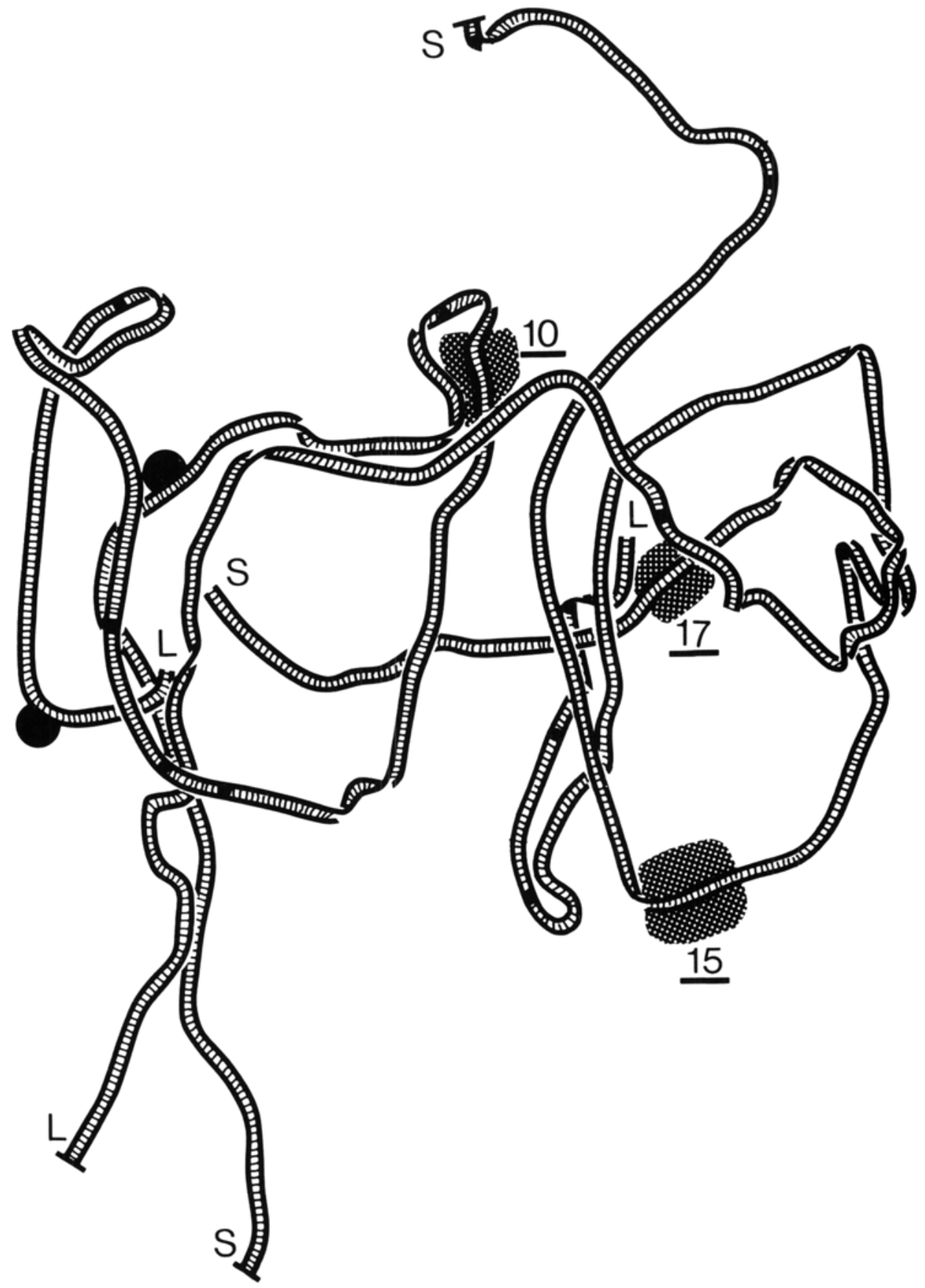

Figure 25. A reconstruction of bivalents 10,15 and 17 at pachytene.

The telomeres of the long arms of bivalents 10 and 15 and the short arm of bivalent 17 are free in the nucleoplasm. A knob is present in both arms of bivalent 10. (The signatures are as described in the legend to Figure 13.) 


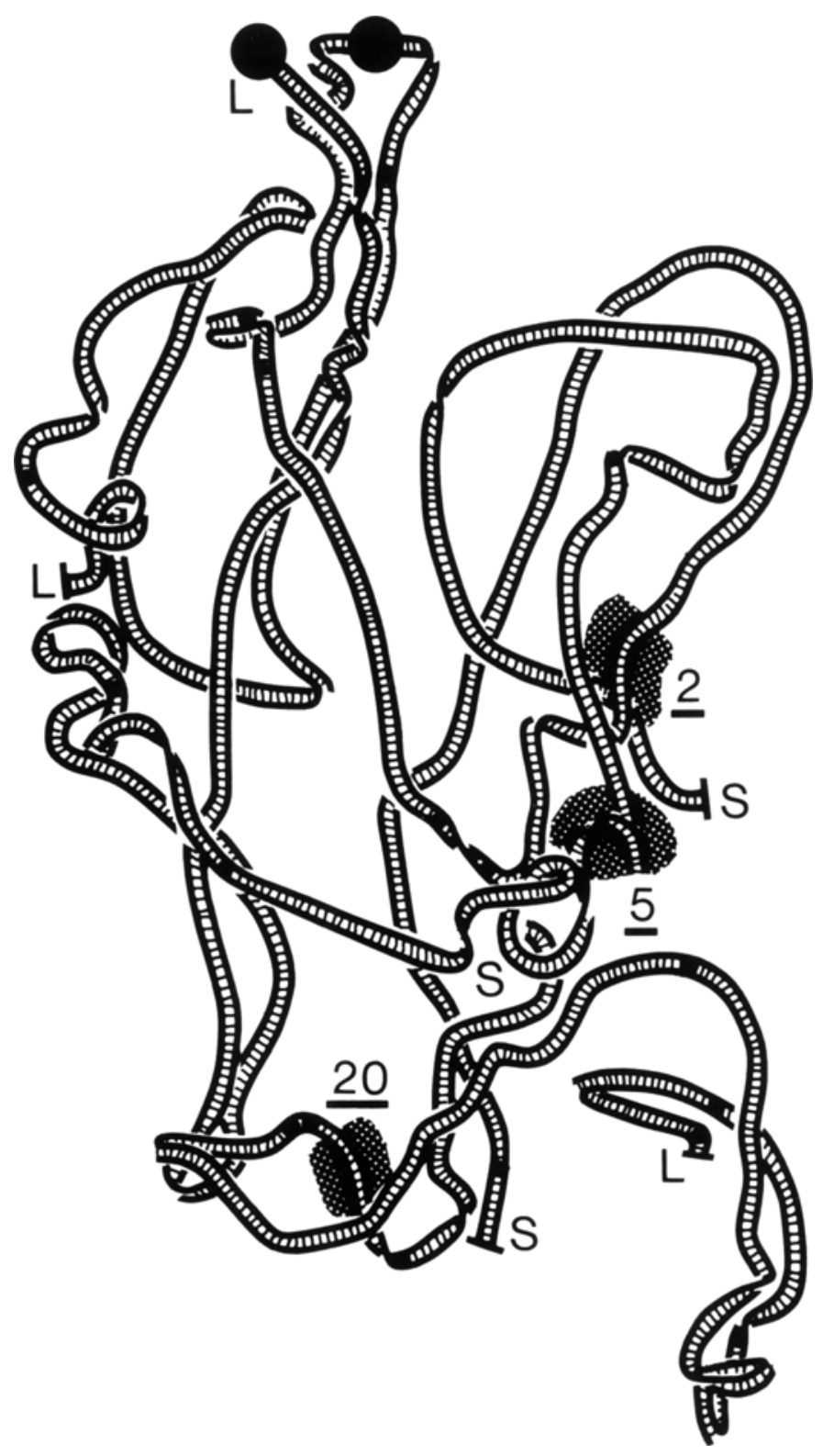

Figure 26. A reconstruction of bivalents 2,5 and 20 at pachytene.

The telomere of the short arm of bivalent 20 is free in the nucleoplasm. One knob is present terminally in the long arm of bivalent 5 and one interstitially in bivalent 2. (The signatures are as described in the legend to Figure 13.) 


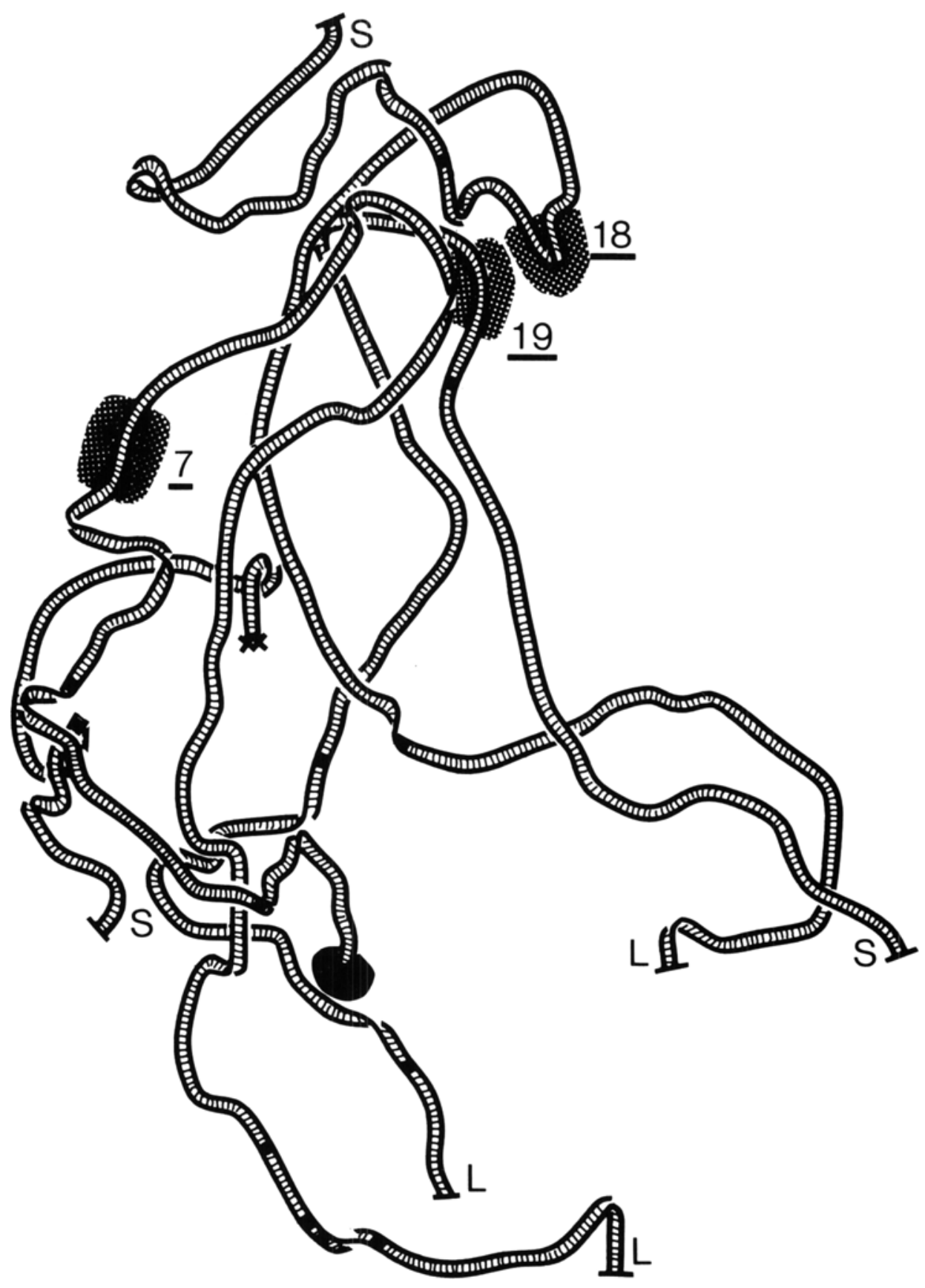

Figure 27. A reconstruction of bivalents 7,18 and 19 at pachytene.

Bivalent 7 is broken within the electron transparent part of the nucleolus organizing region. The distance between the free end of the bivalent segment assumed to constitute the distal part of bivalent 7 and the nucleolus organizing region amounts to $9 \mu \mathrm{m}$. (The signatures are as described in the legend to Figure 13.) 


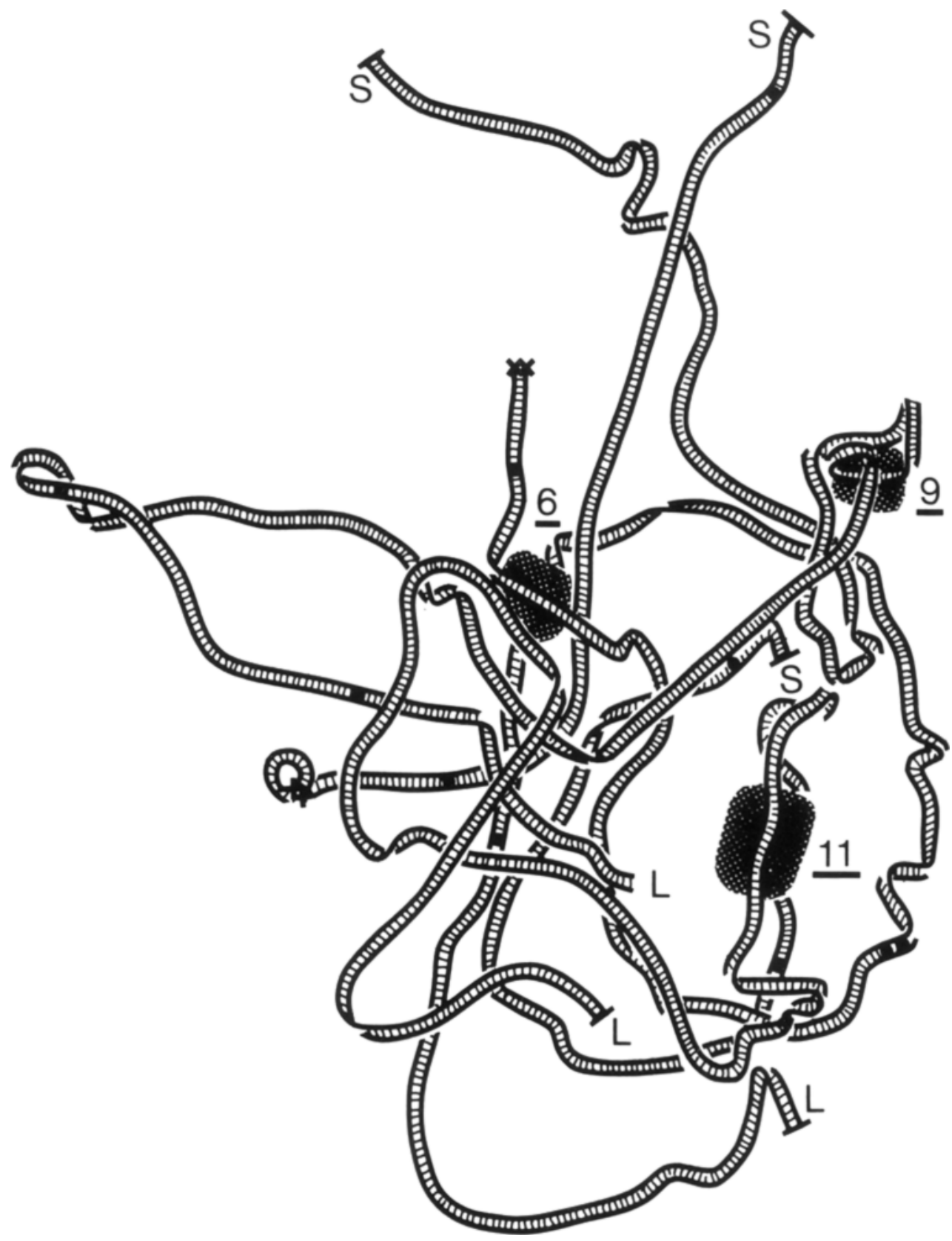

Figure 28. A reconstruction of bivalents 6,9 and 11 at pachytene.

The telomeres of the long arm of bivalent 9 are free in the nucleoplasm. The short arm of bivalent 6 is broken. The distance between the free end of the bivalent segment assumed to constitute the distal part of the short arm of bivalent 6 and the free end of bivalent 6 amounts to $7 \mu \mathrm{m}$. The centromeres of bivalents 11 and 13 (Figure 23) are fused. (The signatures are as described in the legend to Figure 13.) 


\section{REFERENCES}

1. Astaurov, B. L.: Experimental alterations of the developmental cytogenetic mechanisms in mulberry silkworms: Artificial parthenogenesis, polyploidy, gynogenesis and androgenesis. Adv. Morphogenesis 6, 199-257 (1967)

2. Bennett, M. D., G. A. Dover \& R. Riley: Meiotic duration in wheat genotypes with or without homoeologous meiotic chromosome pairing. Proc. R. Soc. Lond. B. 187, 191-207 (1974)

3. Bennett, M. D., J. B. Smith, S. Simpson \& B. WeLLs: Intranuclear fibrillar material in cereal pollen mother cells. Chromosoma (Berl.) 71, 289-332 (1979)

4. Bhowal, J. G.: Nucleolar chromosomes in wheat. Z. Pflanzenzüchtg. 68, 253-257 (1972)

5. Burnham, C. R., J. T. Stout, W. H. WeinhelMeR, R. V. KNowles, \& R. L. Phillips: Chromosome pairing in maize. Genetics 71 , 111-126 (1972)

6. Carpenter, A. T. C.: Synaptonemal complex and recombination nodules in recombinationdeficient mutants of Drosophila melanogaster. Chromosoma (Berl.) 75, 259-292 (1979)

7. Crosby, A. R.: Nucleolar activity of lagging chromosomes in wheat. Am. J. Bot. 44, 813822 (1957)

8. Darvey, N. L. \& C. J. Driscoll: Evidence against somatic association in wheat. Chromosoma (Berl.) 36, 140-149 (1972)

9. Feldman, M.: The effect of chromosomes 5B, 5D and 5A on chromosomal pairing in Triticum aestivum. Proc. Nat. Acad. Sci. USA 55, 14471453 (1966)

10. Feldman, M., T. Mello-Sampayo \& L. Avivi: Somatic association in Triticum aestivum. Proc. Nat. Acad. Sci. USA 56, 1192-1199 (1966)

11. Feldman, M., T. Mello-Sampayo \& L. Avivi: Somatic association of homoeologous chromosomes in Triticum aestivum. Chromosoma (Berl.) 37, 209-222 (1972)

12. Flavell, R. B. \& M. O'Dell: The genetic control of nucleolus formation in wheat. Chromosoma (Berl.) 71, 135-152 (1979)

13. Giluies, C. B.: Ultrastructural analysis of maize pachytene karyotypes by three dimensional reconstruction of the synaptonemal complexes. Chromosoma (Berl.) 43, 145-176 (1973)

14. Giluies, C. B.: An ultrastructural analysis of chromosomal pairing in maize. Compt. Rend. Trav. Lab. Carlsberg 40, 135-161 (1975)

15. HoLm, P. B.: Three dimensional reconstruction of chromosome pairing during the zygotene stage of meiosis in Lilium longiflorum (Thunb.) Carlsberg Res. Commun. 42, 103-151 (1977)
16. Holm, P. B. \& S. W. Rasmussen: Human meiosis I. The human pachytene karyotype analyzed by three dimensional reconstruction of the synaptonemal complex. Carlsberg Res. Commun. 42, 283-323 (1977)

17. Holm, P. B. \& S. W. Rasmussen: Chromosome pairing, recombination nodules and chiasma formation in diploid Bombyx males. Carisberg Res. Commun. 45, 483-548 (1980)

18. Hotta, Y., M. D. Bennett, L. A. Toledo \& H. STERN: Regulation of $\mathrm{R}$ protein and endonuclease activities in meiocytes by homologous chromosome pairing. Chromosoma (Berl.) 72, 191-201 (1979)

19. KawaGuchi, E.: Der Einfluss der Eierbehandlung mit Zentrifugierung auf die Vererbung bei dem Seidenspinner. II. Zytologische Untersuchung bei den polyploiden Seidenspinnern. Cytologia 9, 38-54 (1938)

20. Kinara, H.: Nucleus and chromosome substitution in wheat and Aegilops II. Seiken Zihô 15 , 13-23 (1963)

21. McQuade, H. A. \& B. Bassett: Synaptonemal complexes in premeiotic interphase of pollen mother cells of Triticum aestivum. Chromosoma (Berl.) 63, 153-159 (1977)

22. McQuade, H. A. \& D. G. PICKLEs: Observations on synaptonemal complexes in premeiotic interphase of wheat. Amer. J. Bot. 67, 1361-1373 (1980)

23. Moses, M. J. \& P. A. Poorman: Synaptonemal complex analysis of mouse chromosomal rearrangements. II. Synaptic adjustment in a tandem duplication. Chromosoma (Berl.) 81, 519-535 (1981)

24. Rasmussen, $S$. W.: The meiotic prophase in Bombyx mori females analyzed by three dimensional reconstructions of synaptonemal complexes. Chromosoma (Berl.) 54, 245-293 (1976)

25. Rasmussen. S. W.: Chromosome pairing in triploid females of Bombyx mori analyzed by three dimensional reconstructions of synaptonemal complexes. Carlsberg Res. Commun. 42, 163-197 (1977)

26. RaSMUSSEN, S. W. \& P. B. Holm: Human meiosis II. Chromosome pairing and recombination nodules in human spermatocytes. Carlsberg Res. Commun. 43, 275-327 (1978)

27. Rasmussen, S. W. \& P. B. Holm: Chromosome pairing in autotetraploid Bombyx females. Mechanism for exclusive bivalent formation. Carlsberg Res. Commun. 44, 101-125 (1979)

28. Rasmussen, S. W. \& P. B. Holm: Mechanics of meiosis. Hereditas 93, 187-216 (1980)

29. Rasmussen, S. W. \& P. B. Holm: The meiotic prophase in Bombyx mori. In Insect Ultrastruc- 
ture, H. Akai and R. C. King eds. Plenum Publishing Corporation, New York (1982) in press

30. Riley, R. \& V. Chapman: Genetic control of the cytologically diploid behavior of hexaploid wheat. Nature 182, 713-715 (1958)

31. Riley, R. \& C. Kempanna: The homoeologous nature of the nonhomologous meiotic pairing in Triticum aestivum deficient for chromosome $\mathrm{V}$ (5B). Heredity 18, 287-306 (1963)

32. RiLey, R. \& C. N. LAW: Genetic variation in chromosome pairing. Adv. Genetics 13, 57-114 (1965)

33. Roupakias, D. G. \& P. J. Kaltsikes: Genomic effects on the duration of meiosis in Triticale and its parental species. Can. J. Genet. Cytol. 19. 331-343 (1977)

34. Salema, R. \& I. Brandäo: The use of PIPES buffer in the fixation of plant cells for electron microscopy. J. Submicr. Cytol. 5, 79-96 (1973)

35. Sears, E. R.: The aneuploids of common wheat. Missouri Agr. Exp. Sta. Res. Bull. 572, 1-58 (1954)

36. Sears, E. R.: Genetic control of chromosome pairing in wheat. Ann. Rev. Genet. 10. 31-51 (1976)

37. Westergaard, M. \& D. von Wettstein: The synaptinemal complex. Ann. Rev. Genet. 6, 71110 (1972) 